\title{
STABILITY ESTIMATE FOR THE SEMI-DISCRETE LINEARIZED BENJAMIN-BONA-MAHONY EQUATION*,**
}

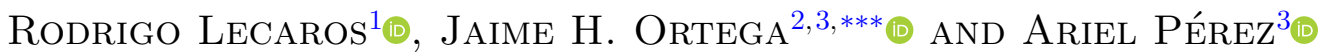

\begin{abstract}
In this work we study the semi-discrete linearized Benjamin-Bona-Mahony equation (BBM) which is a model for propagation of one-dimensional, unidirectional, small amplitude long waves in non-linear dispersive media. In particular, we derive a stability estimate which yields a unique continuation property. The proof is based on a Carleman estimate for a finite difference approximation of Laplace operator with boundary observation in which the large parameter is connected to the mesh size.
\end{abstract}

Mathematics Subject Classification. 35B60, 35L05, 35Q35, 35R45, 65M06.

Received November 26, 2020. Accepted August 29, 2021.

Dedicated to Enrique Zuazua on the occasion of his 60th birthday

\section{INTRODUCTION AND RESULTS}

In this work, we are interested in a linearized version of the Benjamin-Bona-Mahony equation (BBM)

$$
u_{t}+u_{x}+u u_{x}-u_{x x t}=0
$$

proposed by T. Benjamin et al. [2] as a model for propagation of one-dimensional, unidirectional, small amplitude long waves in non-linear dispersive media.

In the last years, several authors have widely studied dispersive equations in the context of controllability and inverse problems. Nevertheless, the BBM equation presents several particularities due to the structure of the operator. In particular, the infinitesimal generator of the semigroup is given by $\left(I-\partial_{x}^{2}\right)^{-1} \partial_{x}$, which is a

* This work is dedicated to Prof. Enrique Zuazua. Dear Enrique, thanks for these years of friendship and for your valuable contributions to the study of control and partial differential equations, and also, for the formation of several generations of researchers in Latin America.

**A. Pérez was founded by the National Agency for Research and Development (ANID)/Scholarship Program/ Doctorado Nacional Chile/2017 - 21170495. R. Lecaros was partially supported by FONDECYT(Chile) Grant 11180874. J.H. Ortega was partially supported by Centro de Modelamiento Matemático (AFB170001) and FONDECYT(Chile) Grant 1201125.

Keywords and phrases: Benjamin-Bona-Mahony equation, unique continuation property, Carleman estimate discrete Carleman inequalities, dispersive equations, water wave equation, finite difference method, semi-discrete equations.

${ }^{1}$ Departamento de Matemática, Universidad Técnica Federico Santa María, Valparaiso, Chile.

2 Centro de Modelamiento Matemático, IRL 2807 CNRS-UChile, Universidad de Chile, Santiago, Chile.

3 Departamento de Ingeniería Matemática, Universidad de Chile, Santiago, Chile.

*** Corresponding author: jortega@dim.uchile.cl 
compact operator, in opposition with the most common situation in PDE where the generator is an unbounded operator among others.

We note some interesting results about the unique continuation property (UCP) for BBM for the continuous case. We refer to the reader to those works and their references for a more detailed discussion: L. Rosier and B.-Y. Zhang in [14] developed a UCP for (1.1) on a periodic domain. Moreover, in [6] P. L. da Silva and I. L. Freire give an alternative proof using geometrical arguments for the periodic case, and for the case when (1.1) is solved in $\mathbb{R}$. In [16], X. Zhang and E. Zuazua considered a linearized BBM equation with space-dependent potential

$$
u_{t}-u_{x x t}=[\alpha(x) u]_{x}+\beta(x) u, \quad(x, t) \in(0,1) \times(0, T) .
$$

In that work, the authors established that the only solution of $(1.2)$, such that $u(0, t)=u(1, t)=0$, is the trivial one $u \equiv 0$ provided that both $\alpha$ and $\beta$ do not vanish on some open subset of $(0,1)$. Furthermore, if $\alpha(x)=-1$ and $\beta(x)=0$ in (1.2), S. Micu proved in [12] a UCP with the additional boundary condition $u_{x}(1, t)=0$, and study controllabily results. On the other hand, in [15], M. Yamamoto established a UCP for BBM-like equation with time and space dependent potential

$$
\partial_{t} u(x, t)-\partial_{x}^{2} \partial_{t} u(x, t)=p(x, t) \partial_{x} u(x, t)+q(x, t) u(x, t), \quad(x, t) \in(0,1) \times(0, T),
$$

where $p \in L^{\infty}((0, T) \times(0,1))$ and $q \in L^{\infty}\left(0, T ; L^{2}(0,1)\right)$. It was shown that the solution of (1.3) shall vanish in $(0,1) \times(0, T)$, provided $u(1, t)=\partial_{x} u(1, t)=0$ for all $t \in(0, T)$ and $u(x, 0)=0$ for $x \in(0,1)$. The main tool to prove this result is a Carleman estimate for the Laplacian operator. Through a more refined version of this Carleman estimate, a stability estimate can be formulated for equation (1.3) (see Sect. 5).

We note that the UCP's have been well studied in several continuous partial differential equations, but it is possible to see that the corresponding discrete case does not holds. For instance, if $u$ is a harmonic function in a domain $\Omega$ and $u=\partial_{n} u=0$ on $\Gamma \subset \partial \Omega$ then $u \equiv 0$ in $\Omega$, it does not generally hold its discrete formulation. We refer to the counterexample due to O. Kavian, presented by E. Zuazua in [18]. However, for (1.4) it is possible to obtain a quantitative UCP under restriction over the mesh size. This result shall be discussed in more detail in Section 1.2.

In this paper, we are interested whether a unique continuation property, as in the work of Yamamoto [15], still holds for a semi-discrete approximation in space of (1.3). In this sense, for $N \in \mathbb{N}$ given, we set the space discretization parameter $h:=1 /(N+1)$. We consider the pairs $\left(x_{i}, t\right)$ with $t \in(0, T), T>0$, and $x_{i}=i h$, for $i=1, \ldots, N$. Thus, the space semi-discrete approximation of equation (1.3) by using the centered finite difference method with respect to the space variable is given by

$$
\partial_{t} u_{i}(t)-\frac{\partial_{t} u_{i+1}(t)-2 \partial_{t} u_{i}(t)+\partial_{t} u_{i-1}(t)}{h^{2}}=p_{i}(t) \frac{u_{i+1}(t)-u_{i-1}(t)}{2 h}+q_{i}(t) u_{i}(t),
$$

for $i \in\{1,2, \ldots, N\}$ and $t \in(0, T)$, where $u_{i}(t)$ stands for $u\left(x_{i}, t\right)$.

\subsection{Notation}

We introduce the notation of meshes and operators that shall be used throughout this paper and necessary to state our results. We consider the following regular partition of the interval $[0,1]$ as

$$
\mathcal{M}_{h}:=\left\{x_{i} \mid x_{i}:=i h, i=0,1, \ldots, N+1\right\},
$$

for $N \in \mathbb{N}$ and $h:=1 /(N+1)$. For any sets of points $\mathcal{W}_{h} \subset \mathcal{M}_{h}$, we define the following dual meshes $\mathcal{W}_{h}^{\prime}$ and $\mathcal{W}_{h}^{*}$ as

$$
\mathcal{W}_{h}^{\prime}:=\tau_{+}\left(\mathcal{W}_{h}\right) \cap \tau_{-}\left(\mathcal{W}_{h}\right), \quad \mathcal{W}_{h}^{*}:=\tau_{+}\left(\mathcal{W}_{h}\right) \cup \tau_{-}\left(\mathcal{W}_{h}\right),
$$




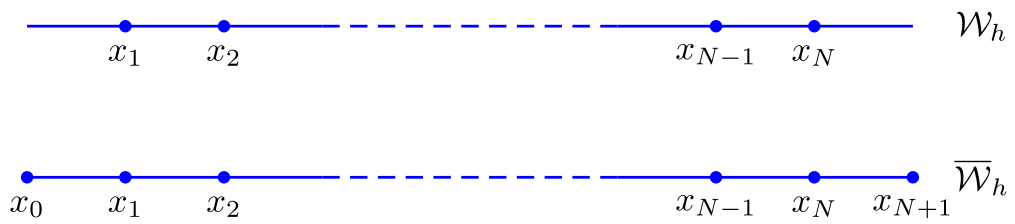

Figure 1. Primal meshes, discretization of space variable.

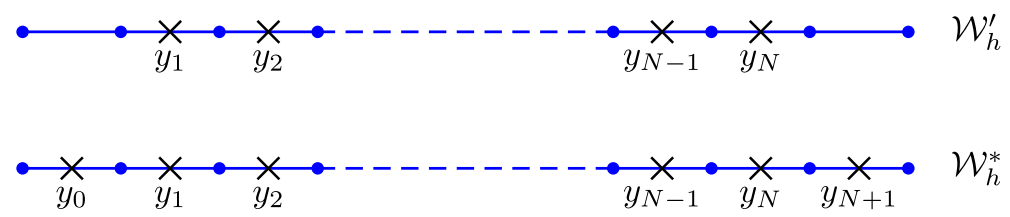

Figure 2. Dual meshes, discretization of space variable.

where

$$
\tau_{ \pm}\left(\mathcal{W}_{h}\right):=\left\{x_{h} \pm \frac{h}{2} \mid x_{h} \in \mathcal{W}_{h}\right\}
$$

We shall denote $\overline{\mathcal{W}}_{h}=\mathcal{W}_{h}^{* *}:=\left(\mathcal{W}_{h}^{*}\right)^{*}$ and $\dot{\mathcal{W}}_{h}=\mathcal{W}_{h}^{\prime \prime}:=\left(\mathcal{W}_{h}^{\prime}\right)^{\prime}$.

We note that if $\dot{\mathcal{W}}_{h}=\mathcal{W}_{h}$, then for two consecutive points $x_{i}, x_{i+1} \in \mathcal{W}_{h}$ we have $x_{i+1}-x_{i}=h$. Thus, any subset $\mathcal{W}_{h} \subset \mathcal{M}_{h}$ that verifies $\stackrel{\circ}{\mathcal{W}}_{h}=\mathcal{W}_{h}$ is called regular mesh. Finally, we define the boundary of a regular mesh $\mathcal{W}_{h}$ as $\partial \mathcal{W}_{h}:=\overline{\mathcal{W}}_{h} \backslash \mathcal{W}_{h}$. These sets defined above can be seen in Figures 1 and 2.

We introduce, using (1.5), the semi-discrete sets. Let us consider $T>0$, we define $Q_{h}:=\mathcal{W}_{h} \times(0, T)$. We also define the dual semi-discrete sets by $Q_{h}^{\prime}:=\mathcal{W}_{h}^{\prime} \times(0, T)$ and $Q_{h}^{*}:=\mathcal{W}_{h}^{*} \times(0, T)$. Similarly, the semi-discrete boundary is given by $\partial Q_{h}=\partial \mathcal{W}_{h} \times(0, T)$.

We define the average operator $A_{h}$ and the difference operator $D_{h}$ by

$$
\begin{aligned}
A_{h}\left(u_{h}\right)\left(x_{h}, t\right) & :=\frac{\tau_{+} u_{h}\left(x_{h}, t\right)+\tau_{-} u_{h}\left(x_{h}, t\right)}{2}, \\
D_{h}\left(u_{h}\right)\left(x_{h}, t\right) & :=\frac{\tau_{+} u_{h}\left(x_{h}, t\right)-\tau_{-} u_{h}\left(x_{h}, t\right)}{h},
\end{aligned}
$$

where $\tau_{ \pm} u_{h}\left(x_{h}, t\right):=u_{h}\left(x_{h} \pm \frac{h}{2}, t\right)$.

We denote by $C\left(Q_{h}\right)$ the set of real-valued functions defined in $Q_{h}$, and by $L_{h}^{2}\left(Q_{h}\right)$ the set $C\left(Q_{h}\right)$ endowed with the norm

$$
\left\|u_{h}\right\|_{L_{h}^{2}\left(Q_{h}\right)}^{2}:=\int_{0}^{T}\|u\|_{L_{h}^{2}\left(\mathcal{W}_{h}\right)}^{2} \mathrm{~d} t
$$

where $\left\|u_{h}\right\|_{L_{h}^{2}\left(\mathcal{W}_{h}\right)}^{2}$ is induced by the inner product

$$
\left\langle u_{h}, v_{h}\right\rangle_{\mathcal{W}_{h}}:=\int_{\mathcal{W}_{h}} u_{h} v_{h}:=h \sum_{x_{h} \in \mathcal{W}_{h}} u_{h}\left(x_{h}\right) v_{h}\left(x_{h}\right) .
$$


For $u_{h} \in C\left(Q_{h}\right)$, we define its $L_{h}^{\infty}\left(Q_{h}\right)$-norm as

$$
\left\|u_{h}\right\|_{L_{h}^{\infty}\left(Q_{h}\right)}:=\max _{\left(x_{h}, t\right) \in Q_{h}}\left\{\left|u_{h}\left(x_{h}, t\right)\right|\right\} .
$$

To introduce the boundary conditions, we define the outward normal for $\left(x_{h}, t\right) \in \partial Q_{h}$ as

$$
n_{h}\left(x_{h}, t\right):=\left\{\begin{aligned}
1 & \left(\tau_{-}\left(x_{h}\right), t\right) \in Q_{h}^{*} \text { and }\left(\tau_{+}\left(x_{h}\right), t\right) \notin Q_{h}^{*}, \\
-1 & \left(\tau_{-}\left(x_{h}\right), t\right) \notin Q_{h}^{*} \text { and }\left(\tau_{+}\left(x_{h}\right), t\right) \in Q_{h}^{*}, \\
0 & \text { otherwise. }
\end{aligned}\right.
$$

We indicate by $\partial Q^{+}\left(\right.$resp. $\left.\partial Q^{-}\right)$the set of points such that $n_{h}\left(x_{h}, t\right)=1\left(\right.$ resp. $\left.n_{h}\left(x_{h}, t\right)=-1\right)$. We also introduce the trace operator for $u_{h} \in C\left(Q_{h}^{*}\right)$ as

$$
\forall\left(x_{h}, t\right) \in \partial Q_{h}, t_{r}\left(u_{h}\right):=\left\{\begin{array}{cl}
\tau_{-} u_{h}\left(x_{h}, t\right) & n_{h}\left(x_{h}, t\right)=1 \\
\tau_{+} u_{h}\left(x_{h}, t\right) & n_{h}\left(x_{h}, t\right)=-1 \\
0 & n_{h}\left(x_{h}, t\right)=0 .
\end{array}\right.
$$

Let us finally introduce the discrete integration on the boundary for $u_{h} \in C\left(\partial \mathcal{W}_{h}\right)$ as

$$
\int_{\partial \mathcal{W}_{h}} u_{h}:=\sum_{x_{h} \in \partial \mathcal{W}_{h}} u_{h}\left(x_{h}\right)
$$

\subsection{Discrete Carleman estimates with boundary observation}

For the discrete Carleman estimate, we consider the weight function of the form $r(x, t)=e^{s \varphi(x, t)}$ for $s \geq 1$, with $\varphi(x, t)=e^{\lambda \psi(x, t)}$ where $\psi$ is a continuous function whose domain of definition $\bar{\Omega}$ is contained in an enlarged smooth open and connected neighborhood $\tilde{\Omega}$. We also assume $\psi \in C^{k}(\bar{\Omega})$ with $k$ large enough such that it satisfies the following property

$$
\partial_{x} \psi(x, t)>0, \quad(x, t) \in \overline{\tilde{\Omega}} \times(0, T) .
$$

The assumption of the higher-order derivatives is needed to obtain the estimates on the weight function presented in Section 4, in contrast to the continuous case. We shall use the same notation for the sample of the continuous function on the discrete or semi-discrete sets. We now state a uniform Carleman estimate for the operator $D_{h}^{2}$ with boundary observation. Although for the Carleman estimate just the condition (1.6) is needed, to achieve the UCP property for the system (1.4) we also consider the following assumption

$$
\partial_{t} \psi(x, t)<0, \quad(x, t) \in \bar{\Omega} \times(0, T) .
$$

It is not difficult to find a function that verifies conditions (1.6) and (1.7), for instance the following function,

$$
\psi(x, t):=\left(x-x_{0}\right)^{2}-t^{2}, x_{0}<0 .
$$

Theorem 1.1. (Discrete Carleman estimate)

Let $\psi$ be a function verifying (1.6) and $T>0$. For the parameter $\lambda_{0} \geq 1$ sufficiently large, there exist $s_{0}\left(\lambda_{0}\right) \geq 1$, $h_{0}>0, \varepsilon_{0}>0$ and $C=C\left(\varepsilon_{0}, s_{0}, \lambda_{0}\right)$ independent of $h>0$ such that 


$$
\begin{array}{r}
C\left(\left\|e^{s \varphi} D_{h}^{2} v_{h}\right\|_{L_{h}^{2}\left(Q_{h}\right)}^{2}+s \int_{\partial Q_{h}^{+}} \varphi \partial_{x} \psi t_{r}\left(e^{2 s \varphi}\right) t_{r}\left(\left|D_{h} v_{h}\right|^{2}\right)+s^{3} \int_{\partial Q_{h}^{+}}\left(\varphi \partial_{x} \psi\right)^{3} t_{r}\left(e^{2 s \varphi}\right) t_{r}\left(A_{h}\left(\left|v_{h}\right|^{2}\right)\right)\right) \\
\geq s^{3}\left\|e^{s \varphi} v_{h}\right\|_{L_{h}^{2}\left(Q_{h}\right)}^{2}+s\left\|e^{s \varphi} D_{h} v_{h}\right\|_{L_{h}^{2}\left(Q_{h}^{*}\right)}^{2},
\end{array}
$$

for all $h \in\left(0, h_{0}\right), s \in\left(s_{0}, \varepsilon_{0} / h\right)$ and $v_{h}$ defined in $Q_{h}:=\dot{\mathcal{M}}_{h} \times(0, T)$.

Several recent works have been concerned with discrete and semi-discrete Carleman estimates for secondorder differential operators. The hyperbolic case has been developed for the one-dimensional case by L. Baudouin and S. Ervedoza [1], to study the stability of an inverse problem to recover a potential term in a semi-discrete wave equation. The elliptic case has been developed by F. Boyer et al. in [3], for the one-dimensional case, to establish a relaxed observability estimate for the associated semi-discrete parabolic equation. In [7], S. Ervedoza and F. de Gournay study the Laplacian operator in arbitrary dimension to prove the stability for the discrete Calderon problem, with limiting Carleman weight function. Semi-discrete Carleman estimates for parabolic operators have been established by F. Boyer and J. Le Rousseau in [4] for multidimensional Cartesian grids. Moreover, in [13], T. N. T. Nguyen studied in the one-dimensional setting a semi-discrete parabolic operator with discontinuous diffusion coefficient; both of them obtain relaxed controllability results for their respective systems.

In the aforementioned works, the discretization was based on a finite difference scheme. Also, the Carleman parameter cannot be arbitrarily large, which is related to the discretization step size, in contrast to the continuous setting. Let us finally mentioned that recently in [10], a fully discrete Carleman estimates for parabolic operator have been obtained by V. Hernández-Santamaría and P. González Casanova, where the spatial and time discrete step-size parameters are connected to the Carleman parameter.

We recall that T. Carleman introduced an estimate, known as the Carleman estimate, to prove a UCP for second-order elliptic partial differential equations in [5] when coefficients fail to be analytic. Nowadays, it has become an efficient tool to prove UCP, the study of controllability, observability, and stabilization for PDEs. We refer to the work of X. Fu et al. [8], and references therein, where the authors present a unified approach to Carleman estimates for second-order PDEs and their applications to control theory and inverse problems.

One of the main difficulties in the development of discrete or semi-discrete Carleman estimates is to compute multiple discrete operators such as $D_{h}$ and $A_{h}$ on the Carleman weight functions. For this reason, we establish Theorem 4.9 (see Sect. 4) to reduce some tedious computation, and it represents an extension of the results presented by F. Boyer et al. in [3] related to discrete estimate on the weight function.

\subsection{Semi-discrete setting and main results}

With the notation we have introduced we can rewrite the semi-discretization (1.4) as

$$
\partial_{t} u_{h}-D_{h}^{2} \partial_{t} u_{h}=p_{h} D_{h} A_{h} u_{h}+q_{h} u_{h} \text { in } Q_{h}:=\dot{\mathcal{M}}_{h} \times(0, T),
$$

where $p_{h}, q_{h} \in L_{h}^{\infty}\left(Q_{h}\right)$. In (1.10) $u_{h}(t)$ provides an approximation of $u\left(x_{h}, t\right), u$ being the solution of the continuous equation (1.3). $\partial_{t} u_{h}$ stands for the first order differentiation with respect to $t$ and the operators $D_{h}$ and $D_{h}^{2}$ are the classical central finite-difference approximation of the space derivatives. We assume that there exists a constant $M>0$ independent of $h$ such that $\max \left\{\left\|p_{h}\right\|_{L_{h}^{\infty}\left(Q_{h}\right)},\left\|q_{h}\right\|_{L_{h}^{\infty}\left(Q_{h}\right)}\right\} \leq M$.

Theorem 1.2. (Stability for space semi-discrete BBM equation)

Let $\psi$ be a function verifying (1.6) and (1.7), and $T>0$. For $\lambda_{0} \geq 1$ sufficiently large, there exist $s_{0}\left(\lambda_{0}, M\right) \geq 1$, $h_{0}>0$ depending on $M, \varepsilon_{0}>0$ and a constant $C>0$ independent of $h>0$ such that the following estimate 
holds

$$
\begin{aligned}
s^{3}\left\|e^{s \varphi} u_{h}\right\|_{L_{h}^{2}\left(Q_{h}\right)}^{2}+s^{3}\left\|e^{s \varphi} \partial_{t} u_{h}\right\|_{L_{h}^{2}\left(Q_{h}\right)}^{2} & +s\left\|e^{s \varphi} D_{h} \partial_{t} u_{h}\right\|_{L_{h}^{2}\left(Q_{h}^{*}\right)}^{2} \\
& \leq C s \int_{\partial Q_{h}^{+}} \varphi \partial_{x} \psi t_{r}\left(e^{2 s \varphi}\right) t_{r}\left(\left|D_{h} \partial_{t} u_{h}\right|^{2}\right) \\
& +C s^{3} \int_{\partial Q_{h}^{+}}\left(\varphi \partial_{x} \psi\right)^{3} t_{r}\left(e^{2 s \varphi}\right) t_{r}\left(A_{h}\left(\left|\partial_{t} u_{h}\right|^{2}\right)\right),
\end{aligned}
$$

for $0<h \leq h_{0}$ and $s \in\left(s_{0}, \varepsilon_{0} / h\right)$ and $u_{h}\left(x_{h}, 0\right)=0$ in $\dot{\mathcal{M}}_{h}$.

As a consequence of Theorem 1.2, we have the following unique continuation property for semi-discrete BBM equation (1.10).

Corollary 1.3. (UCP for a semi-discrete BBM equation)

There exists $h_{0}>0$ depending on $M$ such that if $u_{h}=0$ on $\{1\} \times(0, T), D_{h} u_{h}=0$ on $\{1-h / 2\} \times(0, T)$ and $u_{h}(\cdot, 0)=0$ in $\mathcal{M}_{h}$; then $u_{h}\left(x_{h}, t\right)=0$ in $Q_{h}$ for all $h \in\left(0, h_{0}\right)$.

The methodology of the proof of Theorem 1.2 is similar in spirit to [15], where it have been obtained a UCP for equation (1.3). However, it cannot be followed straightly from the proof of the continuous case since the parameter $s$ cannot be arbitrarily large. As we mentioned above, this parameter is related to the mesh size. Thus, a semi-discrete version of the Carleman estimate used in [15] is not enough. For this reason, we develop a more refined Carleman estimate, (5.2), and its semi-discrete counterpart, see Theorem 1.1.

The rest of this article is organized as follows. First, in Section 2, we introduce and prove some discrete calculus formulas for uniform meshes. Then, we establish a stability estimate for the semi-discrete numerical approximation based on a uniform spatial discretization of equation (1.3) in Section 3. The last three sections are devoted to the proof of the discrete Carleman estimate for a finite-difference approximation of Laplacian operator with boundary observation. In Section 4, we extend the estimations developed in Section 3 of [3] for arbitrary order, which reduce some computation in the proof of the Carleman estimate presented in Section 5. Some calculations are postponed and computed in Section 6.

\section{Calculus formulas for UNiform MeSheS}

In this section, we state the elementary notions concerning discrete calculus formulas. First, we set some useful identities that shall be used in what follows. Then, we present the integration by parts for the difference and average operator. For the sake of presentation, we set the results in the discrete space variable framework instead of the semi-discrete setting.

The following Lemma gives us a calculus rule for finite difference operators. Its proof can be found in [3].

Lemma 2.1. Let $\mathcal{W}_{h} \subset \mathcal{M}_{h}$ be a regular mesh. For $u_{h}, v_{h} \in C\left(\mathcal{M}_{h}\right)$, we have the following identities on $\mathcal{W}_{h}^{*}$

$$
\begin{aligned}
& D_{h}\left(u_{h} v_{h}\right)=D_{h}\left(u_{h}\right) A_{h}\left(v_{h}\right)+A_{h}\left(u_{h}\right) D_{h}\left(v_{h}\right), \\
& A_{h}\left(u_{h} v_{h}\right)=A_{h}\left(u_{h}\right) A_{h}\left(v_{h}\right)+\frac{h^{2}}{4} D_{h}\left(u_{h}\right) D_{h}\left(v_{h}\right) .
\end{aligned}
$$

As a direct consequence of Lemma 2.1, we point out some identities to develop the rest of this paper.

Corollary 2.2. Let $\mathcal{W}_{h} \subseteq \mathcal{M}_{h}$ be a regular mesh.

- For $u \in C\left(\mathcal{W}_{h}\right)$,

$$
A_{h}\left(u_{h}^{2}\right)=\left(A_{h} u_{h}\right)^{2}+\frac{h^{2}}{4}\left(D_{h} u_{h}\right)^{2}, \text { on } \mathcal{W}_{h}^{*}
$$


In particular, for all $u \in C\left(\mathcal{W}_{h}\right)$,

$$
A_{h}\left(u_{h}^{2}\right) \geq\left(A_{h} u_{h}\right)^{2}, \text { on } \mathcal{W}_{h}^{*}
$$

- For $u \in\left(\mathcal{W}_{h}\right)$

$$
D_{h}\left(u_{h}^{2}\right)=2 D_{h} u_{h} A_{h} u_{h}
$$

Note that as a consequence of the first identity in (2.1) of Lemma 2.1, it can be proved by induction the following identity.

Corollary 2.3. Suppose that for $n \in \mathbb{N}$ and $h>0$, the set $(n h / 2,1-n h / 2)$ is not empty. Then, for each $u, v \in C\left(\mathcal{W}_{h}\right)$ we have

$$
D_{h}^{n}(u v)=\sum_{k=0}^{n}\left(\begin{array}{l}
n \\
k
\end{array}\right) D_{h}^{n-k} A_{h}^{k} u A_{h}^{n-k} D_{h}^{k} v \text { on } \mathcal{X}_{h},
$$

where

$$
\mathcal{X}_{h}= \begin{cases}\mathcal{M}_{h}^{*} \cap[n h / 2,1-n h / 2], & \text { if } n \text { is odd } \\ \mathcal{M}_{h} \cap[n h / 2,1-n h / 2], & \text { if } n \text { is even }\end{cases}
$$

Now, we state the following integration by parts presented for discrete space variable. We note that the same identities could be considered in the semi-discrete setting since the temporal variable does not play any significant role.

Proposition 2.4. Let $\mathcal{W}_{h} \subseteq \mathcal{M}_{h}$ be a regular mesh. For $u_{h} \in C\left(\overline{\mathcal{W}}_{h}\right)$ and $v_{h} \in C\left(\mathcal{W}_{h}^{*}\right)$ we have

$$
\int_{\mathcal{W}_{h}} u_{h} D_{h}\left(v_{h}\right)=-\int_{\mathcal{W}_{h}^{*}} D_{h}\left(u_{h}\right) v_{h}+\int_{\partial \mathcal{W}_{h}} u_{h} t_{r}\left(v_{h}\right) n_{h}
$$

and

$$
\int_{\mathcal{W}_{h}} u_{h} A_{h}\left(v_{h}\right)=\int_{\mathcal{W}_{h}^{*}} A_{h}\left(u_{h}\right) v_{h}-\frac{h}{2} \int_{\partial \mathcal{W}_{h}} u_{h} t_{r}\left(v_{h}\right) .
$$

Proof. We have

$$
\begin{aligned}
\int_{\mathcal{W}_{h}} u_{h} \tau_{+}\left(v_{h}\right) & =\int_{\tau_{+}\left(\mathcal{W}_{h}\right)} \tau_{-}\left(u_{h}\right) v_{h} \\
& =\int_{\mathcal{W}_{h}^{*}} \tau_{-}\left(u_{h}\right) v_{h}-\int_{\mathcal{W}_{h}^{*} \backslash \tau_{+}\left(\mathcal{W}_{h}\right)} \tau_{-}\left(u_{h}\right) v_{h} \\
& =\int_{\mathcal{W}_{h}^{*}} \tau_{-}\left(u_{h}\right) v_{h}-\int_{\tau_{-}^{2}\left(\mathcal{W}_{h}\right) \backslash \mathcal{W}_{h}} u_{h} \tau_{+}\left(v_{h}\right) .
\end{aligned}
$$


and

$$
\begin{aligned}
\int_{\mathcal{W}_{h}} u_{h} \tau_{-}\left(v_{h}\right) & =\int_{\tau_{-}\left(\mathcal{W}_{h}\right)} \tau_{+}\left(u_{h}\right) v_{h} \\
& =\int_{\mathcal{W}_{h}^{*}} \tau_{+}\left(u_{h}\right) v_{h}-\int_{\mathcal{W}_{h}^{*} \backslash \tau_{-}\left(\mathcal{W}_{h}\right)} \tau_{+}\left(u_{h}\right) v_{h} \\
& =\int_{\mathcal{W}_{h}^{*}} \tau_{+}\left(u_{h}\right) v_{h}-\int_{\tau_{+}^{2} \mathcal{W}_{h} \backslash \mathcal{W}_{h}} u_{h} \tau_{-}\left(v_{h}\right) .
\end{aligned}
$$

Combining (2.6) and (2.7) it follows that

$$
\begin{aligned}
\int_{\mathcal{W}_{h}} u_{h} D_{h}\left(v_{h}\right)= & -\int_{\mathcal{W}_{h}^{*}} D_{h}\left(u_{h}\right) v_{h}-\frac{1}{h} \int_{\tau_{-}^{2} \mathcal{W}_{h} \backslash \mathcal{W}_{h}} u_{h} \tau_{+}\left(v_{h}\right) \\
& +\frac{1}{h} \int_{\tau_{+}^{2} \mathcal{W}_{h} \backslash \mathcal{W}_{h}} u_{h} \tau_{-}\left(v_{h}\right) \\
& =-\int_{\mathcal{W}_{h}^{*}} D_{h}\left(u_{h}\right) v_{h}+\int_{\partial \mathcal{W}} u_{h} t_{r}\left(v_{h}\right) n_{h} .
\end{aligned}
$$

Similarly, averaging the equations (2.6) and (2.7) we obtain

$$
\begin{aligned}
\int_{\mathcal{W}_{h}} u_{h} A_{h}\left(v_{h}\right)= & \int_{\mathcal{W}_{h}^{*}} A_{h}\left(u_{h}\right) v_{h}-\frac{1}{2} \int_{\tau_{-}^{2}\left(\mathcal{W}_{h}\right) \backslash \mathcal{W}_{h}} u_{h} \tau_{+}\left(v_{h}\right) \\
& -\frac{1}{2} \int_{\tau_{+}^{2} \mathcal{W}_{h} \backslash \mathcal{W}_{h}} u_{h} \tau_{-}\left(v_{h}\right) \\
= & \int_{\mathcal{W}_{h}^{*}} A_{h}\left(u_{h}\right) v_{h}-\frac{h}{2} \int_{\partial \mathcal{W}_{h}} u_{h} t_{r}\left(v_{h}\right),
\end{aligned}
$$

which completes the proof.

Remark 2.5. One can consider the previous result for the meshes $\mathcal{W}_{h}^{\prime}$, see for instance a similar result by $\mathrm{S}$. Ervedoza and F. de Gournay in [7]. In that case we should change the definition of the boundary nodes of our mesh. As we are interested in the boundary that kind of integral-by-parts formulas does not fulfill our goal. In contrast with the integration-by-part formulas from F. Boyer et al. [3], we do not make any distinction on the difference and average operators, then we need to specify that difference in the respective meshes.

\section{Stability estimate For SPACE SEMi-Discrete BMM EQUATION}

This section is devoted to proof Theorem 1.2. We follow as close as possible the ideas from its continuous formulation. For this reason we state a stability estimate for (1.3). The main tool for the proof is a Carleman estimate for Laplacian operator. For sake of exposition we postpone that proof, see Section 5. It is worth to mention that we refined the result presented in [15] (see Sect. 5.1).

\subsection{The continuous case}

We consider $Q:=(0,1) \times(0, T)$, for $T>0$, and we define the classical inner product

$$
(u, v)_{L^{2}(Q)}:=\int_{Q} u v \mathrm{~d} x \mathrm{~d} t
$$


and its respective $L^{2}$-norm $\|u\|_{L^{2}(Q)}^{2}=(u, v)_{L^{2}(Q)}$.

Following the methodology from [15] we can obtain a stability estimate for (1.3). The proof is based on the Carleman estimate (5.2) and Lemma 6.4.2 from V. Isakov [11].

Theorem 3.1. Let $\partial_{x}^{j} \partial_{t}^{k} u \in C([0,1] \times[0, T])$ with $j=0,1,2$ and $k=0,1$. For $\lambda_{0}>0$ sufficiently large, there exist constants $s_{0} \geq 0$ and $C\left(s_{0}, \lambda_{0}, \psi\right)>0$, such that

$$
\begin{aligned}
s^{3}\left\|e^{s \varphi} u\right\|_{L^{2}(Q)}^{2}+s^{3}\left\|e^{s \varphi} \partial_{t} u\right\|_{L^{2}(Q)}^{2}+s\left\|e^{s \varphi} \partial_{x} \partial_{t} u\right\|_{L^{2}(Q)}^{2} \leq & C s^{3} \int_{0}^{T}\left(\left(\partial_{x} \psi\right)^{3} \varphi^{3} e^{2 s \varphi}\left|\partial_{t} u\right|^{2}\right)(1, t) \mathrm{d} t \\
& +C s \int_{0}^{T}\left(\partial_{x} \psi \varphi e^{2 s \varphi}\left|\partial_{x} \partial_{t} u\right|^{2}\right)(1, t) \mathrm{d} t,
\end{aligned}
$$

for all $s \geq s_{0}$, and $u$ verify (1.3) with $u(x, 0)=0$ for all $x \in(0,1)$.

As a Corollary of Theorem 3.1, it follows the main result presented in [15].

Corollary 3.2. Let $\partial_{x}^{j} \partial_{t}^{k} u \in C([0,1] \times[0, T])$ with $0,1,2$ and $k=0,1$. If $u$ is solution of (1.3) such that $u(1, t)=\partial_{x} u(1, t)=0$ for all $t \in(0, T)$ and $u(x, 0)=0$ in $(0,1)$, then $u(x, t)=0$ in $(0,1) \times(0, T)$.

\subsection{Proof of Theorem 1.2}

As we mentioned above the proof of Theorem 1.2 is based on the continuous setting strategy. Then, we write down a space semi-discrete version of Lemma 6.4.2 from [11], which is a Poincaré weighted inequality.

Lemma 3.3. Let $\varphi \in C^{1}\left(\overline{Q_{h}}\right)$ be such that $\frac{\partial \varphi}{\partial t} \leq 0$, then

$$
\int_{Q_{h}}\left|\int_{0}^{t} u_{h}\left(x_{h}, \sigma\right) \mathrm{d} \sigma\right|^{2} e^{2 s \varphi\left(x_{h}, t\right)} \leq T^{2} \int_{Q_{h}}\left(u_{h}\left(x_{h}, t\right)\right)^{2} e^{2 s \varphi\left(x_{h}, t\right)},
$$

for all $u_{h} \in L_{h}^{2}\left(Q_{h}\right)$.

Proof. We have

$$
\begin{aligned}
\int_{0}^{T}\left|\int_{0}^{t} u\left(x_{h}, \sigma\right) \mathrm{d} \sigma\right|^{2} e^{2 s \varphi\left(x_{h}, t\right)} \mathrm{d} t & \leq \int_{0}^{T} t \int_{0}^{t}\left(u\left(x_{h}, \sigma\right)\right)^{2} e^{2 s \varphi\left(x_{h}, t\right)} \mathrm{d} \sigma \mathrm{d} t \\
& =\int_{0}^{T} \int_{\sigma}^{T} t e^{2 s \varphi\left(x_{h}, t\right)}\left(u\left(x_{h}, \sigma\right)\right)^{2} \mathrm{~d} t \mathrm{~d} \sigma \\
& \leq T^{2} \int_{0}^{T}\left(u\left(x_{h}, \sigma\right)\right)^{2} e^{2 s \varphi\left(x_{h}, \sigma\right)} \mathrm{d} \sigma,
\end{aligned}
$$

and integrating over $\dot{\mathcal{M}}_{h}$ we complete the proof.

\subsubsection{Proof of Theorem 1.2}

In this section, we shall give the proof of the Theorem 1.2.

Proof. Note that from (1.10) we have

$$
D_{h}^{2} \partial_{t} u_{h}=\partial_{t} u_{h}-p_{h} D_{h} A_{h} u_{h}-q_{h} u_{h} \text { in } Q_{h} .
$$


Then, applying the Carleman estimate (1.9) to $v_{h}=\partial_{t} u_{h}$ we obtain

$$
\begin{aligned}
s^{3}\left\|e^{s \varphi} \partial_{t} u_{h}\right\|_{L_{h}^{2}\left(Q_{h}\right)}^{2}+s\left\|e^{s \varphi} D_{h} \partial_{t} u_{h}\right\|_{L_{h}^{2}\left(Q_{h}^{*}\right)}^{2} \leq & s \int_{\partial \mathcal{M}_{h}^{+}} \varphi \partial_{x} \psi t_{r}\left(e^{2 s \varphi}\right) t_{r}\left(\left|D_{h} \partial_{t} u_{h}\right|^{2}\right) \\
& +s^{3} \int_{\partial \mathcal{M}_{h}^{+}}\left(\varphi \partial_{x} \psi\right)^{3} t_{r}\left(e^{2 s \varphi}\right) t_{r}\left(A_{h}\left(\left|\partial_{t} u_{h}\right|^{2}\right)\right) \\
& +\left\|e^{s \varphi}\left(\partial_{t} u_{h}-p_{h} D_{h} A_{h} u_{h}-q_{h} u_{h}\right)\right\|_{L_{h}^{2}\left(Q_{h}\right)}^{2},
\end{aligned}
$$

for $0<h \leq h_{0}, s \geq s_{0}$ and $s h<\varepsilon_{0}$.

On the other hand, we note that

$$
D_{h} A_{h} u_{h}\left(x_{h}, t\right)=\int_{0}^{t} D_{h} A_{h} \partial_{t} u_{h}\left(x_{h}, \sigma\right) \mathrm{d} \sigma
$$

and

$$
u_{h}\left(x_{h}, t\right)=\int_{0}^{t} \partial_{t} u_{h}\left(x_{h}, \sigma\right) \mathrm{d} \sigma
$$

since $u_{h}\left(x_{h}, 0\right)=0$ in $\dot{\mathcal{M}}_{h}$. Thus, by Lemma 3.3 it follows that

$$
\begin{array}{rl}
\left\|e^{s \varphi}\left(\partial_{t} u_{h}-p_{h} D_{h} A_{h} u_{h}-q_{h} u_{h}\right)\right\|_{L_{h}^{2}\left(Q_{h}\right) \leq}^{2} & C T^{2}\left\|p_{h}\right\|_{L_{h}^{\infty}\left(Q_{h}\right)}^{2}\left\|e^{s \varphi} D_{h} A_{h} \partial_{t} u_{h}\right\|_{L_{h}^{2}\left(Q_{h}\right)}^{2} \\
& +C\left(1+T^{2}\left\|q_{h}\right\|_{L_{h}^{\infty}\left(Q_{h}\right)}^{2}\right)\left\|e^{s \varphi} \partial_{t} u_{h}\right\|_{L_{h}^{2}\left(Q_{h}\right)}^{2} \\
\leq & C T^{2} M^{2}\left\|e^{s \varphi} D_{h} A_{h} \partial_{t} u_{h}\right\|_{L_{h}^{2}\left(Q_{h}\right)}^{2} \\
& +C\left(1+T^{2} M^{2}\right)\left\|e^{s \varphi} \partial_{t} u_{h}\right\|_{L_{h}^{2}\left(Q_{h}\right)}^{2} .
\end{array}
$$

Now, we focus on the first term of the right-hand side above. Using (2.3) and a discrete integration by parts for the discrete average operator we get

$$
\begin{aligned}
\left\|e^{s \varphi} D_{h} A_{h} \partial_{t} u_{h}\right\|_{L_{h}^{2}\left(Q_{h}\right)}^{2} & =\int_{Q_{h}} e^{2 s \varphi}\left(D_{h} A_{h} \partial_{t} u_{h}\right)^{2} \\
& \leq \int_{Q_{h}} e^{2 s \varphi} A_{h}\left(\left(D_{h} \partial_{t} u_{h}\right)^{2}\right) \\
& =\int_{Q_{h}^{*}} A_{h}\left(e^{2 s \varphi}\right)\left(D_{h} \partial_{t} u_{h}\right)^{2}-\frac{h}{2} \int_{\partial Q_{h}} e^{2 s \varphi} t_{r}\left(\left(D_{h} \partial_{t} u_{h}\right)^{2}\right) \\
& \leq \int_{Q_{h}^{*}} A_{h}\left(e^{2 s \varphi}\right)\left(D_{h} \partial_{t} u_{h}\right)^{2} .
\end{aligned}
$$

From Proposition 4.5 we have $A_{h}\left(e^{2 s \varphi}\right) \leq C_{\lambda} e^{2 s \varphi}$, we thus obtain

$$
\left\|e^{s \varphi} D_{h} A_{h} \partial_{t} u_{h}\right\|_{L_{h}^{2}\left(Q_{h}\right)}^{2} \leq C_{\lambda}\left\|e^{s \varphi} D_{h} \partial_{t} u_{h}\right\|_{L_{h}^{2}\left(Q^{*}\right)} .
$$


Combining (3.2), (3.3) and (3.4) we get

$$
\begin{aligned}
s^{3}\left\|e^{s \varphi} \partial_{t} u_{h}\right\|_{L_{h}^{2}\left(Q_{h}\right)}^{2}+s\left\|e^{s \varphi} D_{h} \partial_{t} u_{h}\right\|_{L_{h}^{2}\left(Q_{h}^{*}\right)}^{2} \leq & s \int_{\partial Q_{h}^{+}} \varphi \partial_{x} \psi t_{r}\left(e^{2 s \varphi}\right) t_{r}\left(\left|D_{h} \partial_{t} u_{h}\right|^{2}\right) \\
& +s^{3} \int_{\partial Q_{h}^{+}}\left(\varphi \partial_{x} \psi\right)^{3} t_{r}\left(e^{2 s \varphi}\right) t_{r}\left(A_{h}\left(\left|\partial_{t} u_{h}\right|^{2}\right)\right) \\
& +\left(1+T^{2} M^{2}\right)\left\|e^{s \varphi} \partial_{t} u_{h}\right\|_{L_{h}^{2}\left(Q_{h}\right)}^{2} \\
& +C_{\lambda} T^{2} M^{2}\left\|e^{s \varphi} D_{h} \partial_{t} u_{h}\right\|_{L_{h}^{2}\left(Q_{h}^{*}\right)}^{2}
\end{aligned}
$$

We note that by choosing some $s \geq T^{2 / 3}\left(1+M^{2}\right)^{1 / 3}+T^{2} M$, the last term on the right-hand side from (3.5) can be absorbs by its left-hand side. Thus, recalling the hypothesis on $s$ from the Carleman estimates, by choosing $s_{1}:=\max \left\{s_{0}, k(M, T)\right\} \geq s_{0}$ large enough we obtain

$$
\begin{aligned}
s^{3}\left\|e^{s \varphi} \partial_{t} u_{h}\right\|_{L_{h}^{2}\left(Q_{h}\right)}^{2}+s\left\|e^{s \varphi} D_{h} \partial_{t} u_{h}\right\|_{L_{h}^{2}\left(Q_{h}^{*}\right)}^{2} \leq & s \int_{\partial Q_{h}^{+}} \varphi \partial_{x} \psi t_{r}\left(e^{2 s \varphi}\right) t_{r}\left(\left|D_{h} \partial_{t} u_{h}\right|^{2}\right) \\
& +s^{3} \int_{\partial Q_{h}^{+}}\left(\varphi \partial_{x} \psi\right)^{3} t_{r}\left(e^{2 s \varphi}\right) t_{r}\left(A_{h}\left(\left|\partial_{t} u_{h}\right|^{2}\right)\right)
\end{aligned}
$$

provided $s \geq s_{1}$, where $k(M, T):=T^{2 / 3}\left(1+M^{2}\right)^{1 / 3}+T^{2} M^{2}$. Now, we need to connect the condition over the Carleman parameter $s$ with the mesh size $h$. Defining

$$
h_{1}:=\frac{\varepsilon_{0}}{s_{1}}
$$

it follows that for $0<h \leq \min \left\{h_{0}, h_{1}\right\}$ we have $s h \leq \varepsilon_{0}$ provided $s \in\left(s_{1}, \varepsilon_{0} / h\right)$, which conclude the proof.

As a consequence, we obtain the UCP presented in Corollary 1.3 for semi-discrete BBM equation (1.10).

\section{Some PRELIminary DiscRete CALCUlus RESUlts}

In this section, we establish some previous estimates for the Carleman weight function that shall be used in the next section to obtain the discrete Carleman estimate, see Theorem 1.1. Recall that our weight function is defined as $e^{s \varphi}$ for $s \geq 1$, with $\varphi=e^{\lambda \psi}$, where $\psi \in C^{k}$ for $k$ sufficiently large and $\lambda \geq 1$. Our goal is to generalize the results presented previously in Section 3, obtained by F. Boyer et al. in [3], related to discrete operations performed on the Carleman weight functions, considering estimates and expansions for higher order discrete operators.

For easier comparison, we use the same notation by setting $r=e^{s \varphi}$ and $\rho=r^{-1}$, these positive parameters $s$ and $h$ shall be large and small respectively and limited by the condition $s h \leq 1$. The proofs are similar in spirit to those given in [3].

We denote by $\mathcal{O}_{\lambda}(s h)$ the functions that verify $\left\|\mathcal{O}_{\lambda}(s h)\right\|_{L^{\infty}\left(Q_{h}\right)} \leq C_{\lambda} s h$ for some constant $C_{\lambda}$ depending on $\lambda$. By $\mathcal{O}(1)$ we denote bounded functions and by $\mathcal{O}_{\lambda}(1)$ a bounded function once $\lambda$ is fixed.

We say that $\alpha$ is a multi-index if $\alpha=\left(\alpha_{1}, \alpha_{2}, \ldots, \alpha_{n}\right) \in \mathbb{N}^{n}$ and for $y \in \mathbb{R}^{n}$ we write:

$$
|\alpha|=\alpha_{1}+\alpha_{2}+\cdots+\alpha_{n}, \quad \partial^{\alpha}=\partial_{x_{1}}^{\alpha_{1}} \ldots \partial_{x_{n}}^{\alpha_{n}}, \quad y^{\alpha}=y_{1}^{\alpha_{1}} \ldots y_{n}^{\alpha_{n}} .
$$


Proposition 4.1. Let us consider $n \in \mathbb{N}$. Let $f$ be $a(n+2)$-times differentiable and $g$ a twice differentiable functions on $\mathbb{R}$. Then

$$
\begin{aligned}
A_{h}^{n} g & =g+R_{A_{h}^{n}}(g), \\
D_{h}^{n} f & =f^{(n)}+R_{D_{h}^{n}}(f) .
\end{aligned}
$$

where $R_{D_{h}^{n}}$ and $R_{A_{h}^{n}}$ are given by

$$
R_{D_{h}^{n}}(f):=h^{2} \sum_{k=0}^{n}\left(\begin{array}{l}
n \\
k
\end{array}\right)(-1)^{k}\left(\frac{(n-2 k)}{2}\right)^{n+2} \int_{0}^{1} \frac{(1-\sigma)^{n+1}}{(n+1) !} f^{(n+2)}\left(\cdot+\frac{(n-2 k) h}{2} \sigma\right) \mathrm{d} \sigma
$$

and

$$
R_{A_{h}^{n}}(g):=\frac{h^{2}}{2^{n+2}} \sum_{k=0}^{n}\left(\begin{array}{l}
n \\
k
\end{array}\right)(n-2 k)^{2} \int_{0}^{1}(1-\sigma) g^{(2)}\left(\cdot+\frac{(n-2 k) h}{2} \sigma\right) \mathrm{d} \sigma .
$$

Proof. The proof of this proposition follows from Taylor expansion

$$
g(x+y)=\sum_{j=0}^{i-1} \frac{y^{j}}{j !} h^{(j)}(x)+y^{i} \int_{0}^{1} \frac{(1-\sigma)^{i-1}}{(i-1) !} g^{(i)}(x+\sigma y) \mathrm{d} \sigma .
$$

First, we use (4.1) with $i=2$ and $y=\frac{(n-2 k) h}{2}$ to obtain

$$
\tau_{+}^{n-2 k} g=g+\frac{(n-2 k) h}{2} g^{\prime}+\left(\frac{(n-2 k) h}{2}\right)^{2} \int_{0}^{1}(1-\sigma) g^{(2)}\left(\cdot+\frac{(n-2 k) h}{2} \sigma\right) \mathrm{d} \sigma .
$$

Then, it follows that

$$
\begin{aligned}
A_{h}^{n} g & =\frac{1}{2^{n}}\left(\tau_{+}+\tau_{-}\right)^{n} g \\
& =\frac{1}{2^{n}} \sum_{k=0}^{n}\left(\begin{array}{l}
n \\
k
\end{array}\right) \tau_{+}^{n-k} \tau_{-}^{k} g \\
& =\frac{1}{2^{n}} \sum_{k=0}^{n}\left(\begin{array}{l}
n \\
k
\end{array}\right) \tau_{+}^{n-2 k} g \\
& =\frac{1}{2^{n}} \sum_{k=0}^{n}\left(\begin{array}{l}
n \\
k
\end{array}\right)\left(g+\frac{(n-2 k) h}{2} g^{\prime}\right)+R_{A^{n}}(g)
\end{aligned}
$$

Now, using $\sum_{k=0}^{n}\left(\begin{array}{l}n \\ k\end{array}\right)=2^{n}$ and $\sum_{k=0}^{n}\left(\begin{array}{l}n \\ k\end{array}\right) k=n 2^{n-1}$ we write

$$
A_{h}^{n} g=g+R_{A_{h}^{n}}(g) .
$$


On the other hand, applying (4.1) for $f$ with $i=n+2$ and $y=\frac{(n-2 k) h}{2}$, we have

$$
\tau_{+}^{n-2 k} f=\sum_{j=0}^{n+1} \frac{1}{j !}\left(\frac{(n-2 k) h}{2}\right)^{j} f^{(j)}+\left(\frac{(n-2 k) h}{2}\right)^{n+2} \int_{0}^{1} \frac{(1-\sigma)^{n+1}}{(n+1) !} f^{(n+2)}\left(\cdot+\frac{(n-2 k) h}{2} \sigma\right) \mathrm{d} \sigma .
$$

Thus, for the difference operator we get

$$
\begin{aligned}
D_{h}^{n} f= & \frac{1}{h^{n}}\left(\tau_{+}-\tau_{-}\right)^{n} f \\
= & \frac{1}{h^{n}} \sum_{k=0}^{n}\left(\begin{array}{l}
n \\
k
\end{array}\right)(-1)^{k} \tau_{+}^{n-k} \tau_{-}^{k} f \\
= & \frac{1}{h^{n}} \sum_{k=0}^{n}\left(\begin{array}{l}
n \\
k
\end{array}\right)(-1)^{k} \tau_{+}^{n-2 k} f \\
= & \frac{1}{h^{n}} \sum_{k=0}^{n}\left(\begin{array}{l}
n \\
k
\end{array}\right)(-1)^{k} \sum_{j=0}^{n+1} \frac{1}{j !}\left((n-2 k) \frac{h}{2}\right)^{j} f^{(j)} \\
& +R_{D_{h}^{n}}(f) \\
= & \frac{1}{h^{n}} \sum_{j=0}^{n+1} \frac{1}{j !} h^{j} \sum_{k=0}^{n}\left(\begin{array}{l}
n \\
k
\end{array}\right)(-1)^{k}\left(\frac{n}{2}-k\right)^{j} f^{(j)}+R_{D_{h}^{n}(f)}
\end{aligned}
$$

Now, using $\sum_{k=0}^{n}(-1)^{k}\left(\begin{array}{l}n \\ k\end{array}\right)(x-k)^{n}=n !$ and $\sum_{k=0}^{n}(-1)^{k}\left(\begin{array}{l}n \\ k\end{array}\right)\left(\frac{n}{2}-k\right)^{n+1}=0$, we obtain

$$
\begin{aligned}
D_{h}^{n} f= & \frac{1}{h^{n}} \frac{1}{(n) !} h^{n} \sum_{k=0}^{n}\left(\begin{array}{l}
n \\
k
\end{array}\right)(-1)^{k}\left(\frac{n}{2}-k\right)^{n} f^{(n)} \\
& +\frac{1}{h^{n}} \frac{1}{(n+1) !} h^{n+1} \sum_{k=0}^{n}\left(\begin{array}{l}
n \\
k
\end{array}\right)(-1)^{k}\left(\frac{n}{2}-k\right)^{n+1} f^{(n+1)}+R_{D^{n}}(f) \\
= & f^{(n)}+R_{D_{h}^{n}}(f),
\end{aligned}
$$

and the proof is complete.

Corollary 4.2. Let $f$ be a $(n+4)$-times differentiable function defined on $\mathbb{R}$ and $m, n \in \mathbb{N}$. Then

$$
A_{h}^{m} D_{h}^{n} f=f^{(n)}+R_{A_{h}^{m}}\left(f^{(n)}\right)+R_{D_{h}^{n}}(f)+R_{A_{h}^{m} D_{h}^{n}}(f),
$$

where

$$
R_{A_{h}^{m} D_{h}^{n}}(f):=\sum_{k, k^{\prime}=0}^{n, m} a_{k, k^{\prime}} \int_{0}^{1} \int_{0}^{1} \frac{(1-\sigma)^{n+1}}{(n+1) !}\left(1-\sigma^{\prime}\right) f^{(n+4)}\left(x+(n-2 k) \frac{h}{2} \sigma+\frac{\left(m-2 k^{\prime}\right) h}{2} \sigma^{\prime}\right) \mathrm{d} \sigma^{\prime} \mathrm{d} \sigma
$$

with

$$
a_{k, k^{\prime}}:=\frac{h^{4}}{2^{m}}\left(\begin{array}{l}
m \\
k^{\prime}
\end{array}\right)\left(\begin{array}{l}
n \\
k
\end{array}\right)(-1)^{k}\left(\frac{(n-2 k)}{2}\right)^{n+2} \frac{\left(m-2 k^{\prime}\right)^{2}}{4} .
$$


Proof. It is enough to see that

$$
\begin{aligned}
A_{h}^{m}\left(D_{h}^{n} f\right) & =A_{h}^{m} f^{(n)}+A_{h}^{m}\left(R_{D_{h}^{n}}(f)\right) \\
& =f^{(n)}+R_{A_{h}^{m}}\left(f^{(n)}\right)+R_{D_{h}^{n}}(f)+R_{A_{h}^{m} D_{h}^{n}}(f) .
\end{aligned}
$$

Note that $R_{A_{h}^{m} D_{h}^{n}}=R_{A_{h}^{m}} \circ R_{D_{h}^{n}}=R_{D_{h}^{n}} \circ R_{A_{h}^{m}}$. Now, we consider two fundamental estimates for our weight function. The proofs of these results can be found in [3]. We consider $\alpha=\left(\alpha_{t}, \alpha_{x}\right) \in \mathbb{N}^{2}$ multi-indices.

Lemma 4.3. Let $\alpha$ and $\beta$ be multi-indices. We have

$$
\begin{aligned}
\partial^{\beta}\left(r \partial^{\alpha} \rho\right)= & |\alpha|^{|\beta|}(-s \varphi)^{|\alpha|} \lambda^{|\alpha+\beta|}\left(\partial_{x} \psi\right)^{\alpha+\beta} \\
& +|\alpha||\beta|(s \varphi)^{|\alpha|} \lambda^{|\alpha+\beta|-1} \mathcal{O}(1)+s^{|\alpha|-1}|\alpha|(|\alpha|-1) \mathcal{O}_{\lambda}(1) \\
= & \mathcal{O}_{\lambda}\left(s^{|\alpha|}\right) .
\end{aligned}
$$

Moreover, let $\sigma \in[0,1]$ and sh $\leq 1$, then $\partial^{\beta}\left(r(x)\left(\partial^{\alpha} \rho\right)(x+\sigma h)\right)=s^{|\alpha|} \mathcal{O}_{\lambda}(1)$.

Corollary 4.4. Let $\alpha, \beta$ and $\delta$ be multi-indices. We have

$$
\begin{aligned}
\partial^{\delta}\left(r^{2}\left(\partial^{\alpha} \rho\right) \partial^{\beta} \rho\right)= & |\alpha+\beta|^{|\delta|}(-s \varphi)^{|\alpha+\beta|} \lambda^{|\alpha+\beta+\delta|}\left(\partial_{x} \psi\right)^{\alpha+\beta+\delta} \\
& +|\delta||\alpha+\beta|(s \varphi)^{|\alpha+\beta|} \lambda^{|\alpha+\beta+\delta|-1} \mathcal{O}(1) \\
& +s^{|\alpha+\beta|-1}(|\alpha|(|\alpha|-1)+|\beta|(|\beta|-1)) \mathcal{O}_{\lambda}(1) \\
= & \mathcal{O}_{\lambda}\left(s^{|\alpha+\beta|}\right) .
\end{aligned}
$$

Corollary 4.2 and Lemma 4.3 yield.

Proposition 4.5. Let $\alpha$ be a multi-index and $n, m \in \mathbb{N}$. Provided sh $\leq 1$, we have

$$
r A_{h}^{m} D_{h}^{n} \partial^{\alpha} \rho=r \partial_{x}^{n} \partial^{\alpha} \rho+s^{|\alpha|+n} \mathcal{O}_{\lambda}\left((s h)^{2}\right)=s^{|\alpha|+n} \mathcal{O}_{\lambda}(1) .
$$

Proof. From Corollary 4.2 we write

$$
r A_{h}^{m} D_{h}^{n} \partial^{\alpha} \rho=r \partial_{x}^{n} \partial^{\alpha} \rho+r R_{A_{h}^{m}}\left(\partial_{x}^{n} \partial^{\alpha} \rho\right)+r R_{D_{h}^{n}}\left(\partial^{\alpha} \rho\right)+r R_{A_{h}^{m} D_{h}^{n}}\left(\partial^{\alpha} \rho\right)
$$

By Lemma 4.3 we have

$$
r(x)\left(\partial_{x}^{n+2} \partial^{\alpha} \rho\right)(x+(n-2 k) h \sigma / 2)=\mathcal{O}_{\lambda}\left(s^{|\alpha|+n+2}\right)
$$

and

$$
r(x) \partial_{x}^{n+4} \partial^{\alpha} \rho(x+(n-2 k) h \sigma / 2)=\mathcal{O}_{\lambda}\left(s^{|\alpha|+n+4}\right) .
$$

Then

$$
\begin{aligned}
r R_{A_{h}^{m}}\left(\partial_{x}^{n} \partial^{\alpha} \rho\right) & =s^{|\alpha|+n} \mathcal{O}_{\lambda}\left((s h)^{2}\right), \\
r R_{D_{h}^{n}}\left(\partial^{\alpha} \rho\right) & =s^{|\alpha|+n} \mathcal{O}_{\lambda}\left((s h)^{2}\right), \\
r R_{A_{h}^{m} D_{h}^{n}}\left(\partial^{\alpha} \rho\right) & =s^{|\alpha|+n} \mathcal{O}_{\lambda}\left((s h)^{4}\right),
\end{aligned}
$$


which yields the result.

Lemma 4.6. Let $\alpha$ and $\beta$ multi-index and $n \in \mathbb{N}$. Provided sh $\leq 1$, we have

$$
A_{h}^{m} D_{h}^{n}\left(\partial^{\beta}\left(r \partial^{\alpha} \rho\right)\right)=\partial_{x}^{n} \partial^{\beta}\left(r \partial^{\alpha} \rho\right)+h^{2} \mathcal{O}_{\lambda}\left(s^{|\alpha|}\right)
$$

Let $\sigma \in[0,1]$, we have $A_{h}^{m} D_{h}^{n} \partial^{\beta}\left(r(x) \partial^{\alpha} \rho(x+\sigma h)\right)=\mathcal{O}_{\lambda}\left(s^{|\alpha|}\right)$.

Proof. By Corollary 4.2 we write

$$
\begin{aligned}
A_{h}^{m} D_{h}^{n}\left(\partial^{\beta}\left(r \partial^{\alpha} \rho\right)\right)= & \partial_{x}^{n} \partial^{\beta}\left(r \partial^{\alpha} \rho\right)+R_{A_{h}^{m}}\left(\partial_{x}^{n} \partial^{\beta}\left(r \partial^{\alpha} \rho\right)\right) \\
& +R_{D_{h}^{n}}\left(\partial^{\beta}\left(r \partial^{\alpha} \rho\right)\right)+R_{A_{h}^{m} D_{h}^{n}}\left(\partial^{\beta}\left(r \partial^{\alpha} \rho\right)\right) .
\end{aligned}
$$

By Lemma 4.3, it follows that

$$
\begin{aligned}
\left(\partial_{x}^{n} \partial^{\beta}\left(r \partial^{\alpha} \rho\right)\right)(x+(n-2 k) h \sigma / 2) & =\mathcal{O}_{\lambda}\left(s^{|\alpha|}\right), \\
\left(\partial_{x}^{n+2} \partial^{\beta}\left(r \partial^{\alpha} \rho\right)\right)(x+(n-2 k) h \sigma / 2) & =\mathcal{O}_{\lambda}\left(s^{|\alpha|}\right), \\
\left(\partial_{x}^{n+4} \partial^{\beta}\left(r \partial^{\alpha} \rho\right)\right)(x+(n-2 k) h \sigma / 2) & =\mathcal{O}_{\lambda}\left(s^{|\alpha|}\right),
\end{aligned}
$$

which concludes the proof of the first result.

On the other hand, we set $\nu(x, \sigma h):=r(x) \rho(x+\sigma h)$ and $\mu_{\alpha}:=r \partial^{\alpha} \rho$. Since $r \rho=1$ it follows that $r(x) \partial^{\alpha} \rho(x+$ $\sigma h)=\nu(x, \sigma h) \mu_{\alpha}(x+\sigma h)$. Note that, by continuous Leibniz rule, $\partial_{x}^{n} \partial^{\beta}\left(\nu \mu_{\alpha}\right)$ is a linear combination of terms of the form $\partial^{\beta^{\prime}} \nu \partial^{\beta^{\prime \prime}} \mu_{\alpha}$, with $\beta^{\prime}+\beta^{\prime \prime}=n+\beta$ and by Lemma 4.3 we write $\partial^{\beta^{\prime}} \nu=\mathcal{O}_{\lambda}(1)$ and $\partial^{\beta^{\prime \prime}} \mu_{\alpha}=\mathcal{O}_{\lambda}\left(s^{|\alpha|}\right)$. Besides, it holds for the terms $\partial_{x}^{n+2} \partial^{\beta}\left(\nu \mu_{\alpha}\right)$ and $\partial_{x}^{n+4} \partial^{\beta}\left(\nu \mu_{\alpha}\right)$ as well. Therefore, applying Corollary 4.2 to $\nu \mu_{\alpha}$ we obtain

$$
A_{h}^{m} D_{h}^{n}\left(\partial^{\beta}\left(r(x) \partial^{\alpha} \rho(x+\sigma h)\right)\right)=\mathcal{O}_{\lambda}\left(s^{|\alpha|}\right)+h^{2} \mathcal{O}_{\lambda}\left(s^{|\alpha|}\right)+h^{4} \mathcal{O}_{\lambda}\left(s^{|\alpha|}\right)
$$

Lemma 4.7. Let $\alpha, \beta, \delta$ be multi-indices and $n, m \in \mathbb{N}$. Provided $s h \leq 1$, we have:

$$
\begin{aligned}
A_{h}^{m} D_{h}^{n} \partial^{\delta}\left(r^{2}\left(\partial^{\alpha} \rho\right) \partial^{\beta} \rho\right) & =\partial_{x}^{n} \partial^{\delta}\left(r^{2}\left(\partial^{\alpha} \rho\right) \partial^{\beta} \rho\right)+h^{2} \mathcal{O}_{\lambda}\left(s^{|\alpha|+|\beta|}\right) \\
& =\mathcal{O}_{\lambda}\left(s^{|\alpha|+|\beta|}\right) .
\end{aligned}
$$

Let $\sigma, \sigma^{\prime} \in[0,1]$. We have

$$
A_{h}^{m} D_{h}^{n} \partial^{\delta}\left(r(x)^{2}\left(\partial^{\alpha} \rho(x+\sigma h)\right) \partial^{\beta} \rho\left(x+\sigma^{\prime} h\right)\right)=\mathcal{O}_{\lambda}\left(s^{|\alpha|+|\beta|}\right) .
$$

Proof. Applying Corollary 4.2 to $\partial^{\delta}\left(r^{2}\left(\partial^{\alpha} \rho\right) \partial^{\beta} \rho\right)$ we obtain

$$
\begin{aligned}
A_{h}^{m} D_{h}^{n} \partial^{\delta}\left(r^{2}\left(\partial^{\alpha} \rho\right) \partial^{\beta} \rho\right)= & \partial_{x}^{n} \partial^{\delta}\left(r^{2}\left(\partial^{\alpha} \rho\right) \partial^{\beta} \rho\right)+R_{A_{h}^{m}}\left(\partial_{x}^{n} \partial^{\delta}\left(r^{2}\left(\partial^{\alpha} \rho\right) \partial^{\beta} \rho\right)\right) \\
& +R_{D_{h}^{n}}\left(\partial^{\delta}\left(r^{2}\left(\partial^{\alpha} \rho\right) \partial^{\beta} \rho\right)\right)+R_{A_{h}^{m} D_{h}^{n}}\left(\partial^{\delta}\left(r^{2}\left(\partial^{\alpha} \rho\right) \partial^{\beta} \rho\right)\right) .
\end{aligned}
$$

Then, the first result follows from Corollary 4.4. For the second one, we proceed similarly as the proof of the second result of Lemma 4.6, that is, we apply Corollary 4.2 to $\nu^{2} \mu_{\alpha} \mu_{\beta}$, then we use continuous Leibniz rule and Lemma 4.6 to conclude. 
Lemma 4.8. Let $\alpha$ be a multi-index. For $j, k, m, n \in \mathbb{N}$ and for $s h \leq 1$, we have

$$
A_{h}^{j} D_{h}^{k} \partial^{\alpha}\left(r A_{h}^{m} D_{h}^{n} \rho\right)=\partial_{x}^{k} \partial^{\alpha}\left(r \partial_{x}^{n} \rho\right)+s^{n} \mathcal{O}_{\lambda}\left((s h)^{2}\right)=s^{n} \mathcal{O}_{\lambda}(1) .
$$

Proof. By Corollary 4.2 we write

$$
\partial^{\alpha}\left(r A_{h}^{m} D_{h}^{n} \rho\right)=\partial^{\alpha}\left(r \partial_{x}^{n} \rho\right)+\partial^{\alpha}\left(r R_{A_{h}^{m}}\left(\partial_{x}^{n} \rho\right)\right)+\partial^{\alpha}\left(r\left(R_{D_{h}^{n}}(\rho)\right)+\partial^{\alpha}\left(r R_{A_{h}^{m} D_{h}^{n}}(\rho)\right) .\right.
$$

Then, applying again Corollary 4.2 to the first term of the above expression, we have

$$
\begin{aligned}
A_{h}^{j} D_{h}^{k} \partial^{\alpha}\left(r A_{h}^{m} D_{h}^{n} \rho\right)= & A_{h}^{j} D_{h}^{k} \partial^{\alpha}\left(r \partial_{x}^{n} \rho\right)+A_{h}^{j} D_{h}^{k} \partial^{\alpha}\left(r R_{A_{h}^{m}}\left(\partial_{x}^{n} \rho\right)\right) \\
& +A_{h}^{j} D_{h}^{k} \partial^{\alpha}\left(r\left(R_{D_{h}^{n}}(\rho)\right)+A_{h}^{j} D_{h}^{k} \partial^{\alpha}\left(r R_{A_{h}^{m} D_{h}^{n}}(\rho)\right)\right. \\
= & \partial_{x}^{k} \partial^{\alpha}\left(r \partial_{x}^{n} \rho\right)+R_{A_{h}^{j}}\left(\partial^{\alpha}\left(r \partial_{x}^{n} \rho\right)\right)+R_{D_{h}^{k}}\left(\partial^{\alpha}\left(r \partial_{x}^{n} \rho\right)\right) \\
& +A_{h}^{j} D_{h}^{k} \partial^{\alpha}\left(r\left(R_{D_{h}^{n}}(\rho)\right)+A_{h}^{j} D_{h}^{k} \partial^{\alpha}\left(r R_{A_{h}^{m} D_{h}^{n}}(\rho)\right) .\right.
\end{aligned}
$$

Thus, by Lemma 4.3, we obtain

$$
A_{h}^{j} D_{h}^{k} \partial^{\alpha}\left(r A_{h}^{m} D_{h}^{n} \rho\right)=\partial_{x}^{k} \partial^{\alpha}\left(r \partial_{x}^{n} \rho\right)+s^{n} \mathcal{O}_{\lambda}\left((s h)^{2}\right) .
$$

Theorem 4.9. Let $\alpha, \beta$ be multi-indices and $j, k, l, m, n, p \in \mathbb{N}$. Provided sh $\leq 1$, we have

$$
\begin{aligned}
A_{h}^{p} D_{h}^{l} \partial^{\beta}\left(r^{2} A_{h}^{j} D_{h}^{k}\left(\partial^{\alpha} \rho\right) A_{h}^{m} D_{h}^{n}(\rho)\right) & =\partial_{x}^{l} \partial^{\beta}\left(r^{2} \partial_{x}^{k} \partial^{\alpha} \rho \partial_{x}^{n} \rho\right)+s^{n+k+|\alpha|} \mathcal{O}_{\lambda}\left((s h)^{2}\right) \\
& =s^{n+k+|\alpha|} \mathcal{O}_{\lambda}(1)
\end{aligned}
$$

Proof. We have

$$
\begin{aligned}
A_{h}^{m} D_{h}^{n}(\rho) & =\partial_{x}^{n} \rho+R_{A_{h}^{m}}\left(\partial_{x}^{n} \rho\right)+R_{D_{h}^{n}}(\rho)+R_{A_{h}^{m} D_{h}^{n}}(\rho), \\
A_{h}^{j} D_{h}^{k}\left(\partial^{\alpha} \rho\right) & =\partial_{x}^{k} \partial^{\alpha} \rho+R_{A_{h}^{j}}\left(\partial_{x}^{k} \partial^{\alpha} \rho\right)+R_{D_{h}^{k}}\left(\partial^{\alpha} \rho\right)+R_{A_{h}^{j} D_{h}^{k}}\left(\partial^{\alpha} \rho\right) .
\end{aligned}
$$

By Lemma 4.7 we write

$$
A_{h}^{p} D_{h}^{l} \partial^{\beta}\left(r^{2} A_{h}^{j} D_{h}^{k}\left(\partial^{\alpha} \rho\right) A_{h}^{m} D_{h}^{n}(\rho)\right)=\partial_{x}^{l} \partial^{\beta}\left(r^{2} \partial_{x}^{k} \partial^{\alpha} \rho \partial_{x}^{n} \rho\right)+s^{n+k+|\alpha|} \mathcal{O}_{\lambda}\left((s h)^{2}\right),
$$

which is our claim.

Let us finally mention that the results of this section can be extended for time-dependent case. For instance, if we consider a weight function of the form $r(x, t)=e^{s \theta(t) \varphi(x)}$ then the condition $s h \leq 1$ must be replaced by $\operatorname{sh}\left(\max _{[0, T]} \theta(t)\right) \leq 1$ which implies that $s \theta(t) h \leq 1$.

\section{Discrete Carleman estimate}

In this section, we establish a discrete Carleman estimate with boundary observation for a finite difference approximation of the Laplacian operator in the one-dimensional setting. In order to do so, it is natural to look closer at the continuous version of such estimates. For this purpose, we follow the methodology of A. V. Fursikov and O. Y. Imanuvilov [9] to obtain a Carleman estimate for Laplacian operator in the continuous setting, which is similar to the estimate obtained by Yamamoto in [15]. The main difference with the one in [15] 
to our estimate is that we do not consider a density argument, and we thus obtain a Carleman estimate with boundary observation. Then, following the methodology in [3] we establish a discrete Carleman estimate.

\subsection{The continuous case}

The proof of the following Carleman estimate has two steps. First, we consider the conjugate operator defined by $P_{\varphi} u:=e^{s \varphi} \partial_{x}^{2}\left(e^{-s \varphi} u\right)$. In this case, our Carleman weight function is defined as $e^{s \varphi}$ for $s>0$ with $\varphi=e^{\lambda \psi}$, where $\lambda>0$, and satisfy

$$
\partial_{x} \psi(x, t)>0, \quad(x, t) \in \bar{Q} .
$$

Then, we split $P_{\varphi}$ into the operators $P_{1}$ and $P_{2}$, and it is estimated the scalar product $\left(P_{1} u, P_{2} u\right)_{L^{2}(Q)}$.

Theorem 5.1. (Carleman estimate) Let $\psi \in C\left(\mathbb{R}^{2}\right)$, and for any $t \in(0, T)$ let $\psi(\cdot, t) \in C^{4}(\mathbb{R})$ such that $\partial_{x} \psi(x, t)>0$ for $(x, t) \in \bar{Q}$. For the parameter $\lambda_{0}>0$ sufficiently large, there exists $s_{0}\left(\lambda_{0}\right) \geq 0$, and $C\left(s_{0}, \lambda_{0}, \psi\right)>0$, such that

$$
\begin{aligned}
& C \int_{Q} e^{2 s \varphi}\left|\partial_{x}^{2} v\right|^{2}+s^{3} \lambda_{0}^{3} \int_{0}^{T}\left(\left(\partial_{x} \psi\right)^{3} \varphi^{3} e^{2 s \varphi}|v|^{2}\right)(1, t)+s \lambda_{0} \int_{0}^{T}\left(\partial_{x} \psi \varphi e^{2 s \varphi}\left|\partial_{x} v\right|^{2}\right)(1, t) \\
& \geq s^{3} \lambda_{0}^{4} \int_{Q}\left(\partial_{x} \psi\right)^{4} \varphi^{3} e^{2 s \varphi}|v|^{2}+s \lambda_{0}^{2} \int_{Q}\left(\partial_{x} \psi\right)^{2} \varphi e^{2 s \varphi}\left|\partial_{x} v\right|^{2}
\end{aligned}
$$

for all $s \geq s_{0}$.

Proof. We set $u=e^{s \varphi} v$. Then the conjugate operator can be expanded as follows

$$
P_{\varphi} u=e^{s \varphi} \partial_{x}^{2}\left(e^{-s \varphi}\right) u+2 e^{s \varphi} \partial_{x}\left(e^{-s \varphi}\right) \partial_{x} u+\partial_{x}^{2} u
$$

Adding $-s \partial_{x}^{2}(\varphi) u,(5.3)$ can be written as

$$
P_{\varphi} u-s \partial_{x}^{2}(\varphi) u=P_{1} u+P_{2} u
$$

where $P_{1} u:=\partial_{x}^{2} u+e^{s \varphi} \partial_{x}^{2}\left(e^{-s \varphi}\right) u$ and $P_{2} u:=2 e^{s \varphi} \partial_{x}\left(e^{-s \varphi}\right) \partial_{x} u-s \partial_{x}^{2}(\varphi) u$. Besides, from (5.4), we have

$$
\left\|P_{\varphi} u-s \partial_{x}^{2}(\varphi) u\right\|_{L^{2}(Q)}^{2}=\left\|P_{1} u\right\|_{L^{2}(Q)}^{2}+\left\|P_{2} u\right\|_{L^{2}(Q)}^{2}+2\left(P_{1} u, P_{2} u\right)_{Q}
$$

Note that

$$
\left\|P_{\varphi} u-s \partial_{x}^{2}(\varphi) u\right\|_{L^{2}(Q)}^{2} \leq C_{\varphi}\left(\left\|P_{\varphi} u\right\|_{L^{2}(Q)}^{2}+s^{2}\|u\|_{L^{2}(Q)}^{2}\right)
$$

since $\partial_{x}^{2} \varphi$ is bounded in $Q$. On the other hand, defining $C_{1} u:=\partial_{x}^{2} u, C_{2} u:=e^{s \varphi} \partial_{x}^{2}\left(e^{-s \varphi}\right) u, B_{1} u:=$ $2 e^{s \varphi} \partial_{x}\left(e^{-s \varphi}\right) \partial_{x} u$ and $B_{2} u:=-s \partial_{x}^{2}(\varphi) u$ we have

$$
\left(P_{1} u, P_{2} u\right)_{L^{2}(Q)}=\sum_{i, j=1}^{2}\left(C_{i}, B_{j}\right)_{L^{2}(Q)} .
$$


We note that, integrating by parts in space, (5.7) can be rewritten as

$$
\begin{aligned}
\left(P_{1} u, P_{2} u\right)_{L^{2}(Q)}= & 2 s^{3} \int_{Q}\left(\partial_{x} \varphi\right)^{2} \partial_{x}^{2} \varphi|u|^{2}+\int_{Q} s^{2}\left(\left(\partial_{x}^{2} \varphi\right)^{2}-\left(\partial_{x} \varphi\right)^{2}-\partial_{x} \varphi \partial_{x}^{2} \varphi\right)|u|^{2}-\frac{s}{2} \int_{Q} \partial_{x}^{4}(\varphi)|u|^{2} \\
& +2 s \int_{Q} \partial_{x}^{2}(\varphi)\left|\partial_{x} u\right|^{2}-\left.s \int_{0}^{T} \partial_{x}(\varphi)\left|\partial_{x} u\right|^{2}\right|_{0} ^{1}+\left.\frac{s}{2} \int_{0}^{T} \partial_{x}^{3}(\varphi)|u|^{2}\right|_{0} ^{1} \\
& +\left.\int_{0}^{T}\left(-s^{3}\left(\partial_{x} \varphi\right)^{3}+s^{2} \partial_{x} \varphi \partial_{x}^{2} \varphi\right)|u|^{2}\right|_{0} ^{1}-\left.s \int_{0}^{T} u \partial_{x} u \partial_{x}^{2}(\varphi)\right|_{0} ^{1}
\end{aligned}
$$

Now, using the Young's inequality on the last integral above, we have

$$
\begin{aligned}
\left(P_{1} u, P_{2} u\right)_{L^{2}(Q)} \geq & 2 s^{3} \int_{Q}\left(\partial_{x} \varphi\right)^{2} \partial_{x}^{2} \varphi|u|^{2}+\int_{Q} s^{2}\left(\left(\partial_{x}^{2} \varphi\right)^{2}-\left(\partial_{x} \varphi\right)^{2}-\partial_{x} \varphi \partial_{x}^{2} \varphi\right)|u|^{2}-\frac{s}{2} \int_{Q} \partial_{x}^{4}(\varphi)|u|^{2} \\
& +2 s \int_{Q} \partial_{x}^{2}(\varphi)\left|\partial_{x} u\right|^{2}-\left.s \int_{0}^{T} \partial_{x}(\varphi)\left|\partial_{x} u\right|^{2}\right|_{0} ^{1}+\left.\frac{s}{2} \int_{0}^{T} \partial_{x}^{3}(\varphi)|u|^{2}\right|_{0} ^{1} \\
& -\left.\frac{s}{2} \int_{0}^{T}\left(\partial_{x}^{2} \varphi\right)^{2}|u|^{2}\right|_{0}-\left.\frac{s}{2} \int_{0}^{T}\left(\partial_{x}^{2} \varphi\right)^{2}|u|^{2}\right|_{1}-\left.\frac{s}{2} \int_{0}^{T}\left|\partial_{x} u\right|^{2}\right|_{0}-\left.\frac{s}{2} \int_{0}^{T}\left|\partial_{x} u\right|^{2}\right|_{1} \\
& +\left.\int_{0}^{T}\left(-s^{3}\left(\partial_{x} \varphi\right)^{3}+s^{2} \partial_{x} \varphi \partial_{x}^{2} \varphi\right)|u|^{2}\right|_{0} ^{1}
\end{aligned}
$$

For $\lambda$ large enough, there exist $C_{\lambda_{0}}>0$ and $\lambda_{0}>0$ such that for $\lambda \geq \lambda_{0}$ we obtain

$$
\begin{aligned}
C_{\lambda_{0}}\left(P_{1} u, P_{2} u\right)_{L^{2}(Q)} \geq & s^{3} \lambda^{4} \int_{Q}\left(\partial_{x} \psi\right)^{4} \varphi^{3}|u|^{2}+s^{2} \lambda^{4} \int_{Q}\left(\partial_{x} \psi\right)^{4} \varphi^{2}|u|^{2}-s \lambda^{4} \int_{Q}\left(\partial_{x} \psi\right)^{4} \varphi|u|^{2} \\
& +s \lambda^{2} \int_{Q}\left(\partial_{x} \psi\right)^{2} \varphi\left|\partial_{x} u\right|^{2}-\left.s \lambda \int_{0}^{T} \partial_{x} \psi \varphi\left|\partial_{x} u\right|^{2}\right|_{0} ^{1}+\left.s \lambda^{3} \int_{0}^{T}\left(\partial_{x} \psi\right)^{3} \varphi|u|^{2}\right|_{0} ^{1} \\
& -\left.s^{3} \lambda^{3} \int_{0}^{T}\left(\partial_{x} \psi\right)^{3} \varphi^{3}|u|^{2}\right|_{0} ^{1}+\left.s^{2} \lambda^{3} \int_{0}^{T}\left(\partial_{x} \psi\right)^{3} \varphi^{2}|u|^{2}\right|_{0} ^{1} \\
& -\left.s \int_{0}^{T}\left(\lambda \partial_{x} \psi\right)^{2} \varphi|u|^{2}\right|_{0}-\left.s \int_{0}^{T}\left(\lambda \partial_{x} \psi\right)^{2} \varphi|u|^{2}\right|_{1}
\end{aligned}
$$

Now, if we fix $\lambda=\lambda_{0}$, there exist $C_{s_{0}, \lambda_{0}}>0$ and $s_{0}\left(\lambda_{0}\right)>0$ such that

$$
\begin{aligned}
C_{s_{0}, \lambda_{0}}\left(P_{1} u, P_{2} u\right)_{L^{2}(Q)} \geq & s^{3} \lambda^{4} \int_{Q}\left(\partial_{x} \psi\right)^{4} \varphi^{3}|u|^{2}+s \lambda^{2} \int_{Q}\left(\partial_{x} \psi\right)^{2} \varphi\left|\partial_{x} u\right|^{2} \\
& -\left.s^{3} \lambda^{3} \int_{0}^{T}\left(\partial_{x} \psi\right)^{3} \varphi^{3}|u|^{2}\right|_{0} ^{1}-\left.s \lambda \int_{0}^{T} \partial_{x} \psi \varphi\left|\partial_{x} u\right|^{2}\right|_{0} ^{1},
\end{aligned}
$$


for $s \geq s_{0}$. Note that $\left|e^{s \varphi} \partial_{x} v\right|^{2}=\left|s u \partial_{x} \varphi+\partial_{x} u\right|^{2} \leq C_{\varphi}\left(s^{2}|u|^{2}+\left|\partial_{x} u\right|^{2}\right)$. Thus, from (5.5), (5.6) and (5.8) we obtain for $\lambda$ large enough

$$
\begin{aligned}
C_{\lambda_{0}, s_{0}, \varphi} \int_{Q} e^{2 s \varphi}\left|\partial_{x}^{2} v\right|^{2} \geq & s^{3} \lambda^{4} \int_{Q}\left(\partial_{x} \psi\right)^{4} \varphi^{3} e^{2 s \varphi}|v|^{2}+s \lambda^{2} \int_{Q}\left(\partial_{x} \psi\right)^{2} \varphi\left|\partial_{x} v\right|^{2} \\
& -\left.s^{3} \lambda^{3} \int_{0}^{T}\left(\partial_{x} \psi\right)^{3} \varphi^{3} e^{2 s \varphi}|v|^{2}\right|_{0} ^{1}-\left.s \lambda \int_{0}^{T} \partial_{x} \psi \varphi e^{2 s \varphi}\left|\partial_{x} v\right|^{2}\right|_{0} ^{1},
\end{aligned}
$$

which proves the required result.

Remark 5.2. Note that taking $x_{0}>1$, the observation data in $(5.2)$ can be switched to the point $(0, t)$, for $t \in(0, T)$.

\subsection{Proof of the discrete Carleman estimate}

Now, we establish a discrete Carleman estimate for the discrete operator $D_{h}^{2}$. Note that this is the discrete Laplacian in one-dimensional setting. There are Carleman estimates for this kind of operator (see F. Boyer et al. $[3,4]$ and S. Ervedoza et al. [7]). The main difference respect to our estimate is the fact that we consider boundary observation, due to the choice of the weight function. Indeed, our Carleman weight function is defined as $e^{s \varphi}$ for $s \geq 1$, with $\varphi=e^{\lambda \psi}$ where $\psi \in C^{k}$ for $k$ sufficiently large and $\lambda \geq 1$. We also assume that

$$
\partial_{x} \psi(x, t)>0, \quad(x, t) \in Q
$$

We follow a classical scheme based on conjugating the original operator with a well chosen exponential function.

\subsubsection{Proof Theorem 1.1}

We make the change of variable $u_{h}=e^{s \varphi} v_{h}$. Our first task is to obtain an expression for $P_{h, \varphi}:=$ $e^{s \varphi} D_{h}^{2}\left(e^{-s \varphi} u_{h}\right)$ with the change of variable proposed. By using (2.5), we have

$$
P_{h, \varphi}=e^{s \varphi} D_{h}^{2}\left(e^{-s \varphi}\right) A_{h}^{2} u+2 e^{s \varphi} A_{h} D_{h}\left(e^{-s \varphi}\right) D_{h} A_{h} u_{h}+e^{s \varphi} A_{h}^{2}\left(e^{-s \varphi}\right) D_{h}^{2} u_{h} .
$$

We define the following coefficients $\alpha_{1}:=e^{s \varphi} A_{h}^{2}\left(e^{-s \varphi}\right), \alpha_{2}:=e^{s \varphi} D_{h}^{2}\left(e^{-s \varphi}\right)$ and $\beta_{1}:=e^{s \varphi} A_{h} D_{h}\left(e^{-s \varphi}\right)$. On the other hand, we set

$$
\begin{aligned}
& C_{1} u_{h}:=\alpha_{1} D_{h}^{2} u_{h}, \\
& C_{2} u_{h}:=\alpha_{2} A_{h}^{2} u_{h}, \\
& B_{1} u_{h}:=2 \beta_{1} D_{h} A_{h} u_{h}, \\
& B_{2} u_{h}:=-s\left(\partial_{x}^{2} \varphi\right) u_{h} .
\end{aligned}
$$

Equation (5.10) thus reads $P_{h, \varphi} u_{h}-s\left(\partial_{x}^{2} \varphi\right) u_{h}=P_{1} u_{h}+P_{2} u_{h}$, where

$$
\begin{aligned}
& P_{1} u_{h}:=C_{1} u_{h}+C_{2} u_{h} \\
& P_{2} u_{h}:=B_{1} u_{h}+B_{2} u_{h} .
\end{aligned}
$$

We write

$$
\left\|P_{h, \varphi}-s\left(\partial_{x}^{2} \varphi\right) u_{h}\right\|_{L_{h}^{2}\left(Q_{h}\right)}^{2}=\left\|P_{1} u_{h}\right\|_{L_{h}^{2}\left(Q_{h}\right)}^{2}+\left\|P_{2} u_{h}\right\|_{L_{h}^{2}\left(Q_{h}\right)}^{2}+2\left\langle P_{1} u_{h}, P_{2} u_{h}\right\rangle_{Q_{h}} .
$$


Since $\partial_{x}^{2} \varphi$ is bounded, we have

$$
\left\|P_{h, \varphi}-s\left(\partial_{x}^{2} \varphi\right) u_{h}\right\|_{L_{h}^{2}\left(Q_{h}\right)}^{2} \leq C\left(\left\|P_{\varphi} u_{h}\right\|_{L_{h}^{2}\left(Q_{h}\right)}^{2}+s^{2}\left\|u_{h}\right\|_{L_{h}^{2}\left(Q_{h}\right)}^{2}\right) .
$$

Now, we shall estimate the scalar product

$$
\left\langle P_{1} u_{h}, P_{2} u_{h}\right\rangle_{Q_{h}}=\sum_{i, j=1}^{2}\left\langle C_{i} u_{h}, B_{j} u_{h}\right\rangle_{Q_{h}} .
$$

For each term of (5.13), we obtain the following results.

Lemma 5.3. For $s h \leq 1$, we have

$$
\left\langle C_{1} u_{h}, B_{1} u_{h}\right\rangle_{Q_{h}}=\int_{Q_{h}^{*}} s \lambda^{2} \varphi\left(\partial_{x} \psi\right)^{2}\left|D_{h} u_{h}\right|^{2}+\int_{Q_{h}^{*}} s \lambda \varphi \partial_{x}^{2} \psi\left|D_{h} u_{h}\right|^{2}-X_{1}+Y_{1}
$$

where

$$
X_{1}:=\int_{Q_{h}^{*}} s \mathcal{O}_{\lambda}\left((s h)^{2}\right)\left|D_{h} u_{h}\right|^{2}
$$

and

$$
Y_{1}:=\int_{\partial Q_{h}}\left(-s \lambda \varphi \partial_{x} \psi+s \mathcal{O}_{\lambda}\left((s h)^{2}\right)\right) t_{r}\left(\left|D u_{h}\right|^{2}\right) n_{h}
$$

Lemma 5.4. For $s h \leq 1$, we have

$$
\left\langle C_{1} u_{h}, B_{2} u_{h}\right\rangle_{Q_{h}} \geq \int_{Q_{h}^{*}} s \lambda^{2}\left(\partial_{x} \psi\right)^{2} \varphi\left|D_{h} u_{h}\right|^{2}+\int_{Q_{h}^{*}} s \lambda \varphi \partial_{x}^{2} \psi\left|D_{h} u_{h}\right|^{2}-X_{2}+Y_{2}
$$

where

$$
X_{2}:=\int_{Q_{h}} s \mathcal{O}_{\lambda}(1)\left|u_{h}\right|^{2}+\int_{Q_{h}^{*}} s \mathcal{O}_{\lambda}\left(h^{2}+(s h)^{2}\right)\left|D_{h} u_{h}\right|^{2}
$$

and

$$
Y_{2}:=\int_{\partial Q_{h}} s \mathcal{O}_{\lambda}(1)\left|u_{h}\right|^{2}-\int_{\partial Q_{h}} s^{2} \mathcal{O}_{\lambda}(1)\left|u_{h}\right|^{2}-\int_{\partial Q_{h}} \mathcal{O}_{\lambda}(1) t_{r}\left(\left|D_{h} u_{h}\right|^{2}\right) .
$$

Lemma 5.5. For $s h \leq 1$, we have

$$
\left\langle C_{2} u_{h}, B_{1} u_{h}\right\rangle_{Q_{h}}=3 \int_{Q_{h}} s^{3} \lambda^{4} \varphi^{3}\left(\partial_{x} \psi\right)^{4}\left|u_{h}\right|^{2}+\int_{Q_{h}}(s \lambda \varphi)^{3} \mathcal{O}(1)\left|u_{h}\right|^{2}-X_{3}+Y_{3},
$$

where

$$
X_{3}:=\int_{Q_{h}} s^{2} \mathcal{O}_{\lambda}(1)+s^{3} \mathcal{O}_{\lambda}\left((s h)^{2}\right)\left|u_{h}\right|^{2}-\int_{Q_{h}^{*}} s \mathcal{O}_{\lambda}\left((s h)^{2}\right)\left|D_{h} u_{h}\right|^{2}
$$


and

$$
\begin{aligned}
Y_{3}:= & \int_{\partial Q_{h}}\left(-\left(s \lambda \varphi \partial_{x} \psi\right)^{3}+s^{2} \mathcal{O}_{\lambda}(1)+s^{3} \mathcal{O}_{\lambda}\left((s h)^{2}\right)\right) t_{r}\left(A_{h}\left(\left|u_{h}\right|^{2}\right)\right) n_{h} \\
& -\int_{\partial Q_{h}} s \mathcal{O}_{\lambda}\left((s h)^{2}\right) t_{r}\left(\left|D_{h} u_{h}\right|^{2}\right) n_{h} .
\end{aligned}
$$

Lemma 5.6. For $s h \leq 1$, we have

$$
\left\langle C_{2} u_{h}, B_{2} u_{h}\right\rangle_{Q_{h}} \geq-\int_{Q_{h}} s^{3} \lambda^{4} \varphi^{3}\left(\partial_{x} \psi\right)^{4}\left|u_{h}\right|^{2}+\int_{Q_{h}} s^{3} \lambda^{3} \varphi^{2}\left(\partial_{x} \psi\right)^{2} \partial_{x}^{2} \psi\left|u_{h}\right|^{2}-X_{4}
$$

where

$$
X_{4}:=\int_{Q_{h}}\left(s^{2} \mathcal{O}_{\lambda}(1)+s^{3} \mathcal{O}_{\lambda}\left((s h)^{2}\right)\right)\left|u_{h}\right|^{2}+\int_{Q_{h}} s \mathcal{O}_{\lambda}(s h)\left|u_{h}\right|^{2}+\int_{Q_{h}^{*}} s \mathcal{O}_{\lambda}\left((s h)^{2}\right)\left|D_{h} u_{h}\right|^{2}
$$

and

$$
Y_{4}:=\int_{\partial Q_{h}} s \mathcal{O}_{\lambda}(1)\left|u_{h}\right|^{2}+\int_{\partial Q_{h}} s \mathcal{O}_{\lambda}\left((s h)^{2}\right)\left|u_{h}\right|^{2}+\int_{\partial Q_{h}} s \mathcal{O}_{\lambda}\left((s h)^{2}\right) t_{r}\left(\left|D_{h} u_{h}\right|^{2}\right) n_{h}
$$

The proof of Lemmas 5.3-5.6 can be found in Section 6 .

Combining the aforementioned Lemmas, for $s h \leq 1$ there exist $\lambda_{1} \geq 1$ and $\varepsilon$ small enough such that for $\lambda \geq \lambda_{1}$ and $0<\operatorname{sh} \leq \min \left\{\varepsilon_{1}(\lambda), 1\right\}=\varepsilon_{1}(\lambda)$, there exists a constant $C_{\lambda_{1}, \varepsilon_{1}}>0$ such that

$$
C_{\lambda_{1}, \varepsilon_{1}}\left\langle P_{1} u_{h}, P_{2} u_{h}\right\rangle_{Q_{h}} \geq \int_{Q_{h}^{*}} s\left(\partial_{x} \psi\right)^{2} \varphi\left|D_{h} u_{h}\right|^{2}+\int_{Q_{h}} s^{3} \varphi^{3}\left(\partial_{x} \psi\right)^{4}\left|u_{h}\right|^{2}+\sum_{i=1}^{4} Y_{i}-X_{i} .
$$

Thus, from (5.11), (5.12) and (5.14) we get

$$
\begin{aligned}
C_{\lambda_{1}, \varepsilon_{1}}\left(\left\|P_{h, \varphi}\right\|_{L_{h}^{2}\left(Q_{h}\right)}^{2}+s^{2}\left\|u_{h}\right\|_{L_{h}^{2}\left(Q_{h}\right)}^{2}\right)+\sum_{i=1}^{4} X_{i} \geq & \sum_{i=1}^{4} Y_{i}+s^{3} \int_{Q_{h}} \varphi^{3}\left(\partial_{x} \psi\right)^{4}\left|u_{h}\right|^{2} \\
& +s \int_{Q_{h}^{*}}\left(\partial_{x} \psi\right)^{2} \varphi\left|D_{h} u_{h}\right|^{2} .
\end{aligned}
$$

On the other hand, we have to deal with the boundary terms. To do this, we can estimate separately the right and left boundary observation. Indeed, let us denote by $Y_{i}^{-}$and $Y_{i}^{+}$the left and the right boundary observation of the term $Y_{i}$, respectively. Once $\lambda$ is fixed, for $s$ large enough there exist positive constants $C_{0}$ and $C_{1}$ such that

$$
\begin{aligned}
& C_{0} s \int_{\partial Q_{h}^{-}} \varphi \partial_{x} \psi t_{r}\left(\left|D_{h} u_{h}\right|^{2}\right) n_{h}+C_{0} s^{3} \int_{\partial Q_{h}^{-}}\left(\varphi \partial_{x} \psi\right)^{3} t_{r}\left(A_{h}\left(\left|u_{h}\right|^{2}\right)\right) \leq \sum_{i=1}^{4} Y_{i}^{-}, \\
& \sum_{i=1}^{4} Y_{i}^{+} \leq C_{1} s \int_{\partial Q_{h}^{+}} \varphi \partial_{x} \psi t_{r}\left(\left|D_{h} u_{h}\right|^{2}\right)+C_{1} s^{3} \int_{\partial Q_{h}^{+}}\left(\varphi \partial_{x} \psi\right)^{3} t_{r}\left(A_{h}\left(\left|u_{h}\right|^{2}\right)\right) .
\end{aligned}
$$


Therefore, if we fix $\lambda=\lambda_{1}$, we can choose $\varepsilon_{0}$ and $h_{0}$ sufficiently small, with $0<\varepsilon_{0} \leq \varepsilon_{1}\left(\lambda_{1}\right)$, and $s_{0} \geq 1$ sufficiently large, such that for $s \geq s_{0}, 0<h \leq h_{0}$, and $s h \leq \varepsilon_{0}$ we obtain

$$
\begin{aligned}
C_{\lambda_{1}, \varepsilon_{0}, s_{0}}\left(\left\|P_{h, \varphi}\right\|_{L_{h}^{2}\left(Q_{h}\right)}^{2}\right) & \geq s^{3}\left\|u_{h}\right\|_{L_{h}^{2}\left(Q_{h}\right)}^{2}+s\left\|D_{h} u_{h}\right\|_{L_{h}^{2}\left(Q_{h}^{*}\right)}^{2} \\
& -C_{1} s \int_{\partial Q_{h}^{+}} \varphi \partial_{x} \psi t_{r}\left(\left|D_{h} u_{h}\right|^{2}\right) n_{h}-C_{1} s^{3} \int_{\partial Q_{h}^{+}}\left(\varphi \partial_{x} \psi\right)^{3} t_{r}\left(A_{h}\left(\left|u_{h}\right|^{2}\right)\right) \\
+ & C_{0} s \int_{\partial Q_{h}^{-}} \varphi \partial_{x} \psi t_{r}\left(\left|D_{h} u_{h}\right|^{2}\right)-C_{0} s^{3} \int_{\partial Q_{h}^{-}}\left(\varphi \partial_{x} \psi\right)^{3} t_{r}\left(A_{h}\left(\left|u_{h}\right|^{2}\right)\right) .
\end{aligned}
$$

Finally, we return to the variable $v_{h}$. To this end, we need the following Lemma.

Lemma 5.7. For $s h \leq 1$, we have

$$
\begin{aligned}
s\left\|e^{s \varphi} D_{h} v_{h}\right\|_{L_{h}^{2}\left(Q_{h}^{*}\right) \leq}^{2} \leq & C\left(s\left\|D_{h} u_{h}\right\|_{L_{h}^{2}\left(Q_{h}^{*}\right)}^{2}+s^{3}\left\|u_{h}\right\|_{L_{h}^{2}\left(Q_{h}\right)}^{2}\right)+s^{2} \mathcal{O}(s h) \int_{\partial Q_{h}}\left|u_{h}\right|^{2}, \\
s \int_{\partial Q_{h}^{+}} \varphi \partial_{x} \psi t_{r}\left(\left|D_{h} u_{h}\right|^{2}\right) \leq & C s^{3} \int_{\partial Q_{h}^{+}} \varphi \partial_{x} \psi t_{r}\left(e^{2 s \varphi} A_{h}\left(\left|v_{h}\right|^{2}\right)\right) \\
& +C s \int_{\partial Q_{h}^{+}} \varphi \partial_{x} \psi t_{r}\left(e^{2 s \varphi}\left|D_{h} v_{h}\right|^{2}\right), \\
s^{3} \int_{\partial Q_{h}^{+}}\left(\varphi \partial_{x} \psi\right)^{3} t_{r}\left(A_{h}\left(\left|u_{h}\right|^{2}\right)\right) \leq & s^{3} \int_{\partial Q_{h}^{+}}\left(\varphi \partial_{x} \psi\right)^{3} t_{r}\left(e^{2 s \varphi} A_{h}\left(\left|v_{h}\right|^{2}\right)\right) \\
& +s \mathcal{O}\left((s h)^{2}\right) \int_{\partial Q_{h}^{+}}\left(\varphi \partial_{x} \psi\right)^{3} t_{r}\left(e^{2 s \varphi}\left|D_{h} v_{h}\right|^{2}\right) .
\end{aligned}
$$

For a proof see Section 6 .

Combining (5.15) with Lemma 5.7, we can choose $\tilde{\varepsilon}>0$ and $\tilde{h}>0$ sufficiently small, with $0<\tilde{h} \leq h_{0}, 0<\tilde{\varepsilon} \leq \varepsilon_{0}$, and $\tilde{s}$ sufficiently large, such that for $s \geq \tilde{s}, 0<h \leq \tilde{h}$, and $s h \leq \tilde{\varepsilon}$, we obtain

$$
\begin{aligned}
s^{3}\left\|e^{s \varphi} v_{h}\right\|_{L_{h}^{2}\left(Q_{h}\right)}^{2}+s\left\|e^{s \varphi} D_{h} v_{h}\right\|_{L_{h}^{2}\left(Q_{h}^{*}\right)}^{2} \leq & C_{\tilde{\varepsilon}, \tilde{s}}\left(\left\|e^{s \varphi} D_{h}^{2} v_{h}\right\|_{L_{h}^{2}\left(Q_{h}\right)}^{2}+s \int_{\partial Q_{h}^{+}} \varphi \partial_{x} \psi t_{r}\left(e^{2 s \varphi}\right) t_{r}\left(\left|D_{h} v_{h}\right|^{2}\right)\right. \\
& \left.+s^{3} \int_{\partial Q_{h}^{+}}\left(\varphi \partial_{x} \psi\right)^{3} t_{r}\left(e^{2 s \varphi}\right) t_{r}\left(A_{h}\left(\left|v_{h}\right|^{2}\right)\right)\right)
\end{aligned}
$$

where we have dropped the left boundary observation, and the proof is complete.

\section{Proof of Intermediate Results}

In this section, we shall prove some technical results used in the development of the discrete Carleman estimate. We consider $s h \leq 1$ in the following Lemmas in order to ensure that every Lemma from Section 4 holds. Recall that our Carleman weight function defined as $r(x):=e^{s \varphi(x)}$ for $s \geq 1$, with $\varphi(x)=e^{\lambda \psi(x)}$ where $\psi \in C^{k}$ for $k$ sufficiently large and $\lambda \geq 1$. We denote $\rho:=r^{-1}$ and $\psi$ verifies $\partial_{x} \psi>0$ in $Q_{h}$. The proof we develop in each Lemma is standard in the following sense. We begin rewritten the semi-discrete integral, if necessary, using some identity related to the discrete operators from Corollary 2.2. Then we apply a semidiscrete integration by parts from Proposition 2.4 to identify the leader terms of the Carleman estimate. Finally, thanks to Theorem 4.9, we can obtain the estimate claimed in each Lemma. 


\subsection{Proof of Lemma 5.3}

Recalling the definition of $C_{1}$ and $B_{1}$, and setting $\gamma_{11}:=\beta_{1} \alpha_{1}$ and $I_{11}:=\left\langle C_{1} u_{h}, B_{1} u_{h}\right\rangle_{Q_{h}}$, we write

$$
I_{11}:=\int_{Q_{h}} 2 \gamma_{11} D_{h}^{2} u_{h} D_{h} A_{h} u_{h}
$$

From Corollary 2.2 the semi-discrete integral $I_{11}$ can be rewritten as

$$
I_{11}=\int_{Q_{h}} \gamma_{1} D_{h}\left(\left|D_{h} u_{h}\right|^{2}\right)
$$

Using Proposition 2.4, for $I_{11}$ we obtain

$$
I_{11}=-\int_{Q_{h}^{*}} D_{h}\left(\gamma_{1}\right)\left|D_{h} u_{h}\right|^{2}+\int_{\partial Q_{h}} \gamma_{1} t_{r}\left(\left|D_{h} u_{h}\right|^{2}\right) n_{h}
$$

The proof is completed by showing that

$$
\begin{aligned}
D_{h}\left(\gamma_{1}\right) & =-s \varphi \lambda^{2}\left(\partial_{x} \psi\right)^{2}-s \lambda \varphi \partial_{x}^{2} \psi+s \mathcal{O}_{\lambda}\left((s h)^{2}\right), \\
\gamma_{1} & =-s \lambda \varphi \partial_{x} \psi+s \mathcal{O}_{\lambda}\left((s h)^{2}\right)
\end{aligned}
$$

which follows from Theorem 4.9 and Corollary 4.4.

\subsection{Proof of Lemma 5.4}

Set $I_{12}:=\left\langle C_{1} u_{h}, B_{2} u_{h}\right\rangle_{Q_{h}}$. From the definition of the operators $C_{1}$ and $B_{2}$, we have

$$
I_{12}:=-s \int_{Q_{h}} \partial_{x}^{2} \varphi \alpha_{1} u_{h} D_{h}^{2} u_{h}
$$

A semi-discrete integration by parts, Proposition 2.4, yields

$$
I_{12}=s \int_{Q_{h}^{*}} D_{h}\left(\partial_{x}^{2} \varphi \alpha_{1} u_{h}\right) D_{h} u_{h}-s \int_{\partial Q_{h}} \partial_{x}^{2} \varphi \alpha_{1} u_{h} t_{r}\left(D u_{h}\right) n_{h}:=I_{12}^{(a)}-I_{12}^{(b)} .
$$

Let us focus on $I_{12}^{(a)}$. We note that thanks to Lemma 2.1, $I_{12}^{(a)}$ can be rewritten as

$$
I_{12}^{(a)}=s \int_{Q_{h}^{*}} D_{h}\left(\partial_{x}^{2} \varphi \alpha_{1}\right) A_{h} u_{h} D_{h} u_{h}+s \int_{Q_{h}^{*}} A_{h}\left(\partial_{x}^{2} \varphi \alpha_{1}\right)\left|D_{h} u_{h}\right|^{2}:=I_{12}^{\left(a_{1}\right)}+I_{12}^{\left(a_{2}\right)} .
$$

To estimate the term $I_{12}^{\left(a_{2}\right)}$, due to Lemma 2.1, we write

$$
A_{h}\left(\alpha_{1} \partial_{x}^{2} \varphi\right)=A_{h}\left(\alpha_{1}\right) A_{h}\left(\partial_{x}^{2} \varphi\right)+\frac{h^{2}}{4} D_{h}\left(\alpha_{1}\right) D_{h}\left(\partial_{x}^{2} \varphi\right) .
$$

By using Proposition 4.1 we obtain the following estimates

$$
\begin{aligned}
& A_{h}\left(\partial_{x}^{2} \varphi\right)=\partial_{x}^{2} \varphi+\mathcal{O}_{\lambda}\left(h^{2}\right), \\
& D_{h}\left(\partial_{x}^{2} \varphi\right)=\partial_{x}^{3} \varphi+\mathcal{O}_{\lambda}\left(h^{2}\right) .
\end{aligned}
$$


Moreover, Lemma 4.8 leads to

$$
\begin{aligned}
& A_{h}\left(\alpha_{1}\right)=1+\mathcal{O}_{\lambda}\left((s h)^{2}\right), \\
& D_{h}\left(\alpha_{1}\right)=\mathcal{O}_{\lambda}\left((s h)^{2}\right) .
\end{aligned}
$$

The previous estimates enables us to write (6.1) as

$$
A_{h}\left(\alpha_{1} \partial_{x}^{2} \varphi\right)=\partial_{x}^{2} \varphi+\mathcal{O}_{\lambda}\left(h^{2}+(s h)^{2}\right)=\lambda^{2}\left(\partial_{x} \psi\right)^{2} \varphi+\lambda \varphi \partial_{x}^{2} \psi+\mathcal{O}_{\lambda}\left(h^{2}+(s h)^{2}\right) .
$$

Therefore, $I_{12}^{\left(a_{2}\right)}$ can be estimated as

$$
I_{12}^{\left(a_{2}\right)}=\int_{Q_{h}^{*}} s \lambda^{2}\left(\partial_{x} \psi\right)^{2} \varphi\left|D_{h} u_{h}\right|^{2}+\int_{Q_{h}^{*}} s \lambda \varphi \partial_{x}^{2} \psi\left|D_{h} u_{h}\right|^{2}+\int_{Q_{h}^{*}} s \mathcal{O}_{\lambda}\left(h^{2}+(s h)^{2}\right)\left|D_{h} u_{h}\right|^{2} .
$$

On the other hand, by using $(2.3), I_{12}^{\left(a_{1}\right)}$ can be rewritten as

$$
I_{12}^{\left(a_{1}\right)}=\frac{s}{2} \int_{Q_{h}^{*}} D_{h}\left(\alpha_{1} \partial_{x}^{2} \varphi\right) D_{h}\left(\left|u_{h}\right|^{2}\right) .
$$

A semi-discrete integration by parts with respect to the difference operator $D_{h}$ leads to

$$
I_{12}^{\left(a_{1}\right)}=-\frac{s}{2} \int_{Q_{h}} D_{h}^{2}\left(\partial_{x}^{2} \varphi \alpha_{1}\right)\left|u_{h}\right|^{2}+\frac{s}{2} \int_{\partial Q_{h}} t_{r}\left(D_{h}\left(\partial_{x}^{2} \varphi \alpha_{1}\right)\right)\left|u_{h}\right|^{2} n_{h} .
$$

By using (2.5), it follows that

$$
D_{h}^{2}\left(\partial_{x}^{2} \varphi \alpha_{1}\right)=D_{h}^{2}\left(\partial_{x}^{2} \varphi\right) A_{h}^{2}\left(\alpha_{1}\right)+2 D_{h} A_{h}\left(\partial_{x}^{2} \varphi\right) A_{h} D_{h}\left(\alpha_{1}\right)+A_{h}^{2}\left(\partial_{x}^{2} \varphi\right) D_{h}^{2}\left(\alpha_{1}\right) .
$$

Now, applying Lemma 4.8 to $\alpha_{1}:=e^{s \varphi} A_{h}^{2}\left(e^{-s \varphi}\right)$, we have

$$
\begin{aligned}
A_{h}^{2}\left(\alpha_{1}\right) & =\mathcal{O}_{\lambda}(1), \\
A_{h} D_{h}\left(\alpha_{1}\right) & =\mathcal{O}_{\lambda}(1), \\
D_{h}^{2}\left(\alpha_{1}\right) & =\mathcal{O}_{\lambda}(1) .
\end{aligned}
$$

Moreover, applying Proposition 4.1 to $\partial_{x}^{2} \varphi$, we get

$$
\begin{aligned}
D_{h}^{2}\left(\partial_{x}^{2} \varphi\right) & =\partial_{x}^{4} \varphi+\mathcal{O}_{\lambda}\left(h^{2}\right)=\mathcal{O}_{\lambda}(1), \\
D_{h} A_{h}\left(\partial_{x}^{2} \varphi\right) & =\partial_{x}^{3} \varphi+\mathcal{O}_{\lambda}\left(h^{2}\right)=\mathcal{O}_{\lambda}(1), \\
A_{h}^{2}\left(\partial_{x}^{2} \varphi\right) & =\partial_{x}^{2} \varphi+\mathcal{O}_{\lambda}\left(h^{2}\right)=\mathcal{O}_{\lambda}(1) .
\end{aligned}
$$

Thus, (6.3) can be estimated as

$$
D_{h}^{2}\left(\alpha_{1} \partial_{x}^{2} \varphi\right)=\mathcal{O}_{\lambda}(1)
$$

Similarly, we get

$$
D_{h}\left(\alpha_{1} \partial_{x}^{2} \varphi\right)=\mathcal{O}_{\lambda}(1)
$$


Hence, for $I_{12}^{\left(a_{1}\right)}$ we obtain

$$
I_{12}^{\left(a_{1}\right)}=-s \int_{Q_{h}} \mathcal{O}_{\lambda}(1)\left|u_{h}\right|^{2}+s \int_{\partial Q_{h}} \mathcal{O}_{\lambda}(1)\left|u_{h}\right|^{2}
$$

Finally, by using the Young's inequality, $I_{12}^{(b)}$ can be bounded as

$$
\left|I_{12}^{(b)}\right| \leq s^{2} \int_{\partial Q_{h}}\left|\mathcal{O}_{\lambda}(1)\right|\left|u_{h}\right|^{2}+\int_{\partial Q_{h}}\left|\mathcal{O}_{\lambda}(1)\right| t_{r}\left(\left|D_{h} u_{h}\right|^{2}\right) .
$$

Therefore, collecting the estimates $(6.2),(6.4)$ and $(6.5), I_{12}$ can be estimated as

$$
I_{12} \geq \int_{Q_{h}^{*}} s \lambda^{2}\left(\partial_{x} \psi\right)^{2} \varphi\left|D_{h} u_{h}\right|^{2}+\int_{Q_{h}^{*}} s \lambda \varphi \partial_{x}^{2} \psi\left|D_{h} u_{h}\right|^{2}-X_{2}+Y_{2},
$$

where $X_{2}$ and $Y_{2}$ are given by

$$
X_{2}:=\int_{Q_{h}} s \mathcal{O}_{\lambda}(1)\left|u_{h}\right|^{2}+\int_{Q_{h}^{*}} s \mathcal{O}_{\lambda}\left(h^{2}+(s h)^{2}\right)\left|D_{h} u_{h}\right|^{2}
$$

and

$$
Y_{2}:=\int_{\partial Q_{h}} s \mathcal{O}_{\lambda}(1)\left|u_{h}\right|^{2}-\int_{\partial Q_{h}} s^{2} \mathcal{O}_{\lambda}(1)\left|u_{h}\right|^{2}-\int_{\partial Q_{h}} \mathcal{O}_{\lambda}(1) t_{r}\left(\left|D_{h} u_{h}\right|^{2}\right),
$$

which is our claim.

\subsection{Proof of Lemma 5.5}

Setting $\gamma_{21}:=\alpha_{2} \beta_{1}$ and $I_{21}:=\left\langle C_{2} u_{h}, B_{1} u_{h}\right\rangle_{Q_{h}}$. Let us compute

$$
I_{21}=\int_{Q_{h}} 2 \gamma_{21} A_{h}^{2} u_{h} D_{h} A_{h} u_{h}
$$

By using Lemma 2.1 the above semi-discrete integral can be rewritten as

$$
I_{21}=\int_{Q_{h}} \gamma_{21} D_{h}\left(\left(A_{h} u_{h}\right)^{2}\right) .
$$

A semi-discrete integration by parts with respect to the difference operator yields

$$
\begin{aligned}
I_{21} & =-\int_{Q_{h}^{*}} D_{h}\left(\gamma_{21}\right)\left(A_{h} u_{h}\right)^{2}+\int_{\partial Q_{h}} \gamma_{21} t_{r}\left(\left(A u_{h}\right)^{2}\right) n_{h} \\
& :=I_{21}^{(a)}+I_{21}^{(b)} .
\end{aligned}
$$

Let us first estimate $I_{21}^{(a)}$. Note that (2.2) leads to

$$
I_{21}^{(a)}=-\int_{Q_{h}^{*}} D_{h}\left(\gamma_{21}\right) A_{h}\left(u_{h}^{2}\right)+\frac{h^{2}}{4} \int_{Q_{h}^{*}} D_{h}\left(\gamma_{21}\right)\left|D_{h} u_{h}\right|^{2} .
$$


Then, by Proposition 2.4 we obtain

$$
I_{21}^{(a)}=-\int_{Q_{h}} A_{h} D_{h}\left(\gamma_{21}\right)\left|u_{h}\right|^{2}-\frac{h}{2} \int_{\partial Q} t_{r}\left(D_{h}\left(\gamma_{21}\right)\right)\left|u_{h}\right|^{2}+\frac{h^{2}}{4} \int_{Q_{h}^{*}} D_{h}\left(\gamma_{21}\right)\left|D_{h} u_{h}\right|^{2} .
$$

Recalling that $\alpha_{2}:=e^{s \varphi} D_{h}^{2}\left(e^{-s \varphi}\right)$ and $\beta_{1}:=e^{s \varphi} A_{h} D_{h}\left(e^{-s \varphi}\right)$ we have

$$
A_{h} D_{h}\left(\gamma_{21}\right)=-3 s^{3} \lambda^{4} \varphi^{3}\left(\partial_{x} \psi\right)^{4}+(s \lambda \varphi)^{3} \mathcal{O}(1)+s^{2} \mathcal{O}_{\lambda}(1)+s^{3} \mathcal{O}_{\lambda}\left((s h)^{2}\right)
$$

and $D_{h}\left(\gamma_{21}\right)=s^{3} \mathcal{O}_{\lambda}(1)$, by virtue of Proposition 4.9 and Corollary 4.1. Hence, for $I_{21}^{(a)}$ we obtain the following estimate

$$
\begin{aligned}
I_{21}^{(a)}= & 3 s^{3} \lambda^{4} \int_{Q_{h}} \varphi^{3}\left(\partial_{x} \psi\right)^{4}\left|u_{h}\right|^{2}+\int_{Q_{h}}(s \lambda \varphi)^{3} \mathcal{O}(1)\left|u_{h}\right|^{2}-\int_{Q_{h}}\left(s^{2} \mathcal{O}_{\lambda}(1)+s^{3} \mathcal{O}_{\lambda}\left((s h)^{2}\right)\left|u_{h}\right|^{2}\right. \\
& -\int_{Q_{h}^{*}} s \mathcal{O}_{\lambda}\left((s h)^{2}\right)\left|D_{h} u_{h}\right|^{2}-s^{2} \int_{\partial Q_{h}} \mathcal{O}_{\lambda}(s h)\left|u_{h}\right|^{2} .
\end{aligned}
$$

On the other hand, $I_{21}^{(b)}$ can be estimated as

$$
I_{21}^{(b)}=\int_{\partial Q_{h}}-\left(s \lambda \varphi \partial_{x} \psi\right)^{3}+s^{2} \mathcal{O}_{\lambda}(1)+s^{3} \mathcal{O}_{\lambda}\left((s h)^{2}\right) t_{r}\left(A_{h}\left(\left|u_{h}\right|^{2}\right)\right) n_{h},
$$

since $\gamma_{21}=-\left(s \lambda \varphi \partial_{x} \psi\right)^{3}+s^{2} \mathcal{O}_{\lambda}(1)+s^{3} \mathcal{O}_{\lambda}\left((s h)^{2}\right)$, due to Proposition 4.9. Thus, combining (6.6) with (6.7) and (6.8) the Lemma follows.

\subsection{Proof of Lemma 5.6}

Let $I_{22}:=\left\langle C_{2} u_{h}, B_{2} u_{h}\right\rangle_{Q_{h}}$. By definition of $C_{2}$ and $B_{2}$, let us estimate the semi-discrete integral

$$
I_{22}=-s \int_{Q_{h}} \alpha_{2} \partial_{x}^{2} \varphi A_{h}^{2}\left(u_{h}\right) u_{h}
$$

To this end, by using $(2.2), I_{22}$ can be rewritten as

$$
I_{22}=-s \int_{Q_{h}} \alpha_{2} \partial_{x}^{2} \varphi\left|u_{h}\right|^{2}+\frac{-s h^{2}}{4} \int_{Q_{h}} \alpha_{2} \partial_{x}^{2} \varphi u_{h} D_{h}^{2} u_{h}:=I_{22}^{(a)}+I_{22}^{(b)} .
$$

Since $s h \leq 1$, from Proposition 4.5 and Lemma 4.3 we have for $\alpha_{2}:=e^{s \varphi} D_{h}^{2}\left(e^{-s \varphi}\right)$ the following estimate

$$
\alpha_{2}=(s \lambda \varphi)^{2}\left(\partial_{x} \psi\right)^{2}+s \mathcal{O}_{\lambda}(1)+s^{2} \mathcal{O}_{\lambda}\left((s h)^{2}\right) .
$$

Furthermore, noting that $\partial_{x}^{2} \varphi=\lambda^{2}\left(\partial_{x} \psi\right)^{2} \varphi+\lambda \varphi \partial_{x}^{2} \psi$, with the previous estimate for $\alpha_{2}$ we obtain

$$
\alpha_{2} \partial_{x}^{2} \varphi=s^{2} \lambda^{4} \varphi^{3}\left(\partial_{x} \psi\right)^{4}+s^{2} \lambda^{3} \varphi^{2}\left(\partial_{x} \psi\right)^{2} \partial_{x}^{2} \psi+s \mathcal{O}_{\lambda}(1)+s^{2} \mathcal{O}_{\lambda}\left((s h)^{2}\right)=s^{2} \mathcal{O}_{\lambda}(1)
$$

Then, $I_{22}^{(a)}$ is estimated as

$$
I_{22}^{(a)}=-s^{3} \lambda^{4} \int_{Q_{h}} \varphi^{3}\left(\partial_{x} \psi\right)^{4}\left|u_{h}\right|^{2}-\int_{Q_{h}}\left(-s^{3} \lambda^{3} \varphi^{2}\left(\partial_{x} \psi\right)^{2} \partial_{x}^{2} \psi+s^{2} \mathcal{O}_{\lambda}(1)+s^{3} \mathcal{O}_{\lambda}\left((s h)^{2}\right)\right)\left|u_{h}\right|^{2} .
$$


Similarly, for $I_{22}^{(b)}$, a semi-discrete integration by parts yields

$$
I_{22}^{(b)}=\frac{s h^{2}}{4} \int_{Q_{h}^{*}} D_{h}\left(\alpha_{2} \partial_{x}^{2} \varphi u_{h}\right) D_{h} u_{h}-\frac{s h^{2}}{4} \int_{\partial Q_{h}} \alpha_{2} \partial_{x}^{2} \varphi u_{h} t_{r}\left(D_{h} u_{h}\right) n_{h}:=I_{22}^{\left(b_{1}\right)}-I_{22}^{\left(b_{2}\right)} .
$$

Let us estimate $I_{22}^{\left(b_{2}\right)}$. Note that by using (6.9) and Young's inequality, $I_{22}^{\left(b_{2}\right)}$ can be bounded as

$$
\left|I_{22}^{\left(b_{2}\right)}\right| \leq s \int_{\partial Q_{h}}\left|\mathcal{O}_{\lambda}\left((s h)^{2}\right)\right|\left|u_{h}\right|^{2} n_{h}+s \int_{\partial Q_{h}}\left|\mathcal{O}_{\lambda}\left((s h)^{2}\right)\right| t_{r}\left(\left|D_{h} u_{h}\right|^{2}\right)
$$

Now, let us focus on $I_{22}^{\left(b_{1}\right)}$. Using Lemma 2.1 we write $D_{h}\left(\left|u_{h}\right|^{2}\right)=2 D_{h} u_{h} A_{h} u_{h}$. Thus, $I_{22}^{\left(b_{1}\right)}$ can be written as

$$
I_{22}^{\left(b_{1}\right)}=\frac{s h^{2}}{8} \int_{Q_{h}^{*}} D_{h}\left(\alpha_{2} \partial_{x}^{2} \varphi\right) D_{h}\left(\left|u_{h}\right|^{2}\right)+\frac{s h^{2}}{4} \int_{Q_{h}^{*}} A_{h}\left(\alpha_{2} \partial_{x}^{2} \varphi\right)\left|D_{h} u_{h}\right|^{2} .
$$

We now use a semi-discrete integration by parts on the first integral above to obtain

$$
I_{22}^{\left(b_{1}\right)}=-\frac{s h^{2}}{8}\left(\int_{Q_{h}} D_{h}^{2}\left(\alpha_{2} \partial_{x}^{2} \varphi\right)\left|u_{h}\right|^{2}+\int_{\partial Q_{h}}\left|u_{h}\right|^{2} t_{r}\left(D_{h}\left(\alpha_{2} \partial_{x}^{2} \varphi\right)\right) n_{h}\right)+\frac{s h^{2}}{4} \int_{Q_{h}^{*}} A_{h}\left(\alpha_{2} \partial_{x}^{2} \varphi\right)\left|D_{h} u_{h}\right|^{2} .
$$

To obtain an estimate for $I_{22}^{\left(b_{1}\right)}$ we claim that

$$
\begin{aligned}
A_{h}\left(\alpha_{2} \partial_{x}^{2} \varphi\right) & =s^{2} \mathcal{O}_{\lambda}(1), \\
D_{h}^{2}\left(\alpha_{2} \partial_{x}^{2} \varphi\right) & =s^{2} \mathcal{O}_{\lambda}(1), \\
D_{h}\left(\alpha_{2} \partial_{x}^{2} \varphi\right) & =s^{2} \mathcal{O}_{\lambda}(1) .
\end{aligned}
$$

Indeed, to prove the estimate (6.12) we use Lemma 2.1 to write

$$
A_{h}\left(\alpha_{2} \partial_{x}^{2} \varphi\right)=A_{h}\left(\alpha_{2}\right) A_{h}\left(\partial_{x}^{2} \varphi\right)+\frac{h^{4}}{2} D_{h}\left(\alpha_{2}\right) D_{h}\left(\partial_{x}^{2} \varphi\right)
$$

Then, thanks to Lemma 4.8, we obtain

$$
\begin{aligned}
A_{h}\left(\alpha_{2}\right) & =s^{2} \mathcal{O}_{\lambda}(1), \\
D_{h}\left(\alpha_{2}\right) & =s^{2} \mathcal{O}_{\lambda}(1) .
\end{aligned}
$$

Moreover, using Proposition 4.1 we have

$$
\begin{aligned}
& A_{h}\left(\partial_{x}^{2} \varphi\right)=\partial_{x}^{2} \varphi+h^{2} \mathcal{O}_{\lambda}(1), \\
& D_{h}\left(\partial_{x}^{2} \varphi\right)=\partial_{x}^{3} \varphi+h^{2} \mathcal{O}_{\lambda}(1),
\end{aligned}
$$

and since $\partial_{x}^{2} \varphi=\mathcal{O}_{\lambda}(1),(6.12)$ follows. For the estimate (6.13), applying (2.5) it follows that

$$
D_{h}^{2}\left(\alpha_{2} \partial_{x}^{2} \varphi\right)=D_{h}^{2}\left(\alpha_{2}\right) A_{h}^{2}\left(\partial_{x}^{2} \varphi\right)+2 A_{h} D_{h}\left(\alpha_{2}\right) A_{h} D_{h}\left(\partial_{x}^{2} \varphi\right)+D_{h}^{2}\left(\partial_{x}^{2} \varphi\right) A_{h}^{2}\left(\alpha_{2}\right)
$$


Similarly, by using Lemma 4.8 and Proposition 4.1 we have

$$
\begin{aligned}
D_{h}^{2}\left(\alpha_{2}\right) & =s^{2} \mathcal{O}_{\lambda}(1), A_{h} D_{h}\left(\alpha_{2}\right)=s^{2} \mathcal{O}_{\lambda}(1), A_{h}^{2}\left(\alpha_{2}\right)=s^{2} \mathcal{O}_{\lambda}(1), \\
A_{h}^{2}\left(\partial_{x}^{2} \varphi\right) & =\partial_{x}^{2} \varphi+h^{2} \mathcal{O}_{\lambda}(1), A_{h} D_{h}\left(\partial_{x}^{2} \varphi\right)=\partial_{x}^{3} \varphi+h^{2} \mathcal{O}_{\lambda}(1),
\end{aligned}
$$

These estimates and (6.15) establishes (6.13). The same methodology works for (6.14).

We thus have, from (6.12)-(6.14), the following estimate for $I_{22}^{\left(b_{1}\right)}$

$$
I_{22}^{\left(b_{1}\right)}=-s \int_{Q_{h}} \mathcal{O}_{\lambda}(s h)\left|u_{h}\right|^{2}+s \int_{Q_{h}^{*}} \mathcal{O}_{\lambda, \epsilon}\left((s h)^{2}\right)\left|D_{h} u_{h}\right|^{2}+s \int_{\partial Q_{h}} \mathcal{O}_{\lambda}(1)\left|u_{h}\right|^{2} .
$$

Therefore, combining (6.10) with (6.16) and (6.11) proves the estimate for $I_{22}$.

\subsection{Proof of Lemma 5.7}

We begin proving the first inequality (5.16) of our Lemma. Recalling that $v_{h}=u_{h} e^{-s \varphi}$, thanks to Lemma 2.1 and Young's inequality, we have

$$
\begin{aligned}
\left\|e^{s \varphi} D_{h} v_{h}\right\|_{L_{h}^{2}\left(Q_{h}^{*}\right)}^{2} & \leq\left\|e^{s \varphi} D_{h}\left(u_{h}\right) A_{h}\left(e^{-s \varphi}\right)\right\|_{L_{h}^{2}\left(Q_{h}^{*}\right)}^{2}+\left\|e^{s \varphi} D_{h}\left(e^{-s \varphi}\right) A_{h}\left(u_{h}\right)\right\|_{L_{h}^{2}\left(Q_{h}^{*}\right)}^{2} \\
& :=J_{1}+J_{2},
\end{aligned}
$$

Let us first estimate $J_{2}$. Using (2.3) and a discrete integration by part respect to the average operator we obtain

$$
J_{2}=\int_{Q_{h}} A_{h}\left(\left(e^{s \varphi} D_{h}\left(e^{-s \varphi}\right)\right)^{2}\right)\left|u_{h}\right|^{2}+\frac{h}{2} \int_{\partial Q_{h}} t_{r}\left(\left(e^{s \varphi} D_{h}\left(e^{-s \varphi}\right)\right)^{2}\right)\left|u_{h}\right|^{2} .
$$

Then, by virtue of Proposition $4.5, J_{2}$ can be estimated as follows

$$
J_{2} \leq s \int_{Q_{h}}\left|u_{h}\right|^{2}+s \mathcal{O}_{\lambda}(s h) \int_{Q_{h}}\left|u_{h}\right|^{2} .
$$

It remains to proves that

$$
J_{1} \leq \mathcal{O}_{\lambda}(1) \int_{Q_{h}}\left|D_{h} u\right|^{2}
$$

which it follows from Proposition 4.5, and the proof for (5.16) is complete.

To prove the inequality (5.17), we note that

$$
D_{h} u_{h}(h / 2, t)=D_{h}\left(e^{s \varphi}\right) A_{h} v_{h}(h / 2, t)+D_{h}\left(v_{h}\right) A_{h}\left(e^{s \varphi}\right)(h / 2, t),
$$

due to Lemma 2.1. Hence, Young's inequality and Proposition 4.5 yield

$$
e^{-2 s \varphi}\left|D_{h} u_{h}\right|^{2}(h / 2, t) \leq C_{\lambda}\left(s^{2}\left|A_{h} v_{h}\right|^{2}(h / 2, t)+\left|D_{h} v_{h}\right|^{2}(h / 2, t)\right),
$$

which establishes inequality (5.17).

We proceed similarly for (5.18). From (2.2) we have

$$
A_{h}\left(\left|u_{h}\right|^{2}\right)=\left|A_{h} u_{h}\right|^{2}+\frac{h^{2}}{4}\left|D_{h} u_{h}\right|^{2} .
$$


Repeated application of Lemma 2.1 and Young's inequality lead to

$$
A_{h}\left(\left|u_{h}\right|^{2}\right) \leq C\left(A_{h}\left(v_{h}^{2}\right)\left|A_{h} e^{s \varphi}\right|^{2}+h^{4}\left|D_{h} v_{h}\right|^{2}\left|D_{h} e^{s \varphi}\right|^{2}+h^{2}\left|D_{h} v_{h}\right|^{2}\left|A_{h} e^{s \varphi}\right|^{2}+h^{2}\left|D_{h} e^{s \varphi}\right|^{2}\left|A_{h} v\right|^{2}\right) .
$$

Then, using Proposition 4.5 we obtain

$$
e^{-2 s \varphi} A_{h}\left(u_{h}^{2}\right) \leq\left(\mathcal{O}_{\lambda}(1)+\mathcal{O}_{\lambda}\left((s h)^{2}\right) A_{h}\left(\left|v_{h}\right|^{2}\right)+\left(h^{2}+h^{2} \mathcal{O}_{\lambda}\left((s h)^{2}\right)\right)\left|D_{h} v_{h}\right|^{2},\right.
$$

which completes the proof.

\section{Comments}

The results presented in Section 4 are of independent interest in view of its potential applications on problems related to semi-discrete Carleman estimates. For instance, it could be used to answer the challenge proposed by C. Zheng in [17], that is, to obtain a semi-discrete global Carleman estimates for fourth-order Schrödinger equation and establish a semi-discrete counterpart of the main results presented in that paper. Even in the continuous setting, there are few papers about the stability of an inverse problem for higher-order equations, via Carleman estimates, due to tedious computation and the increased complexity. To our knowledge, there are no results about discrete or semi-discrete Carleman estimates for higher-order operators. Thus, Theorem 4.9 can be a useful tool to obtain results in that direction.

A possible extension of this paper could be to reformulate Theorem 1.2 for some families of non-uniform meshes. The Carleman estimate (1.11) is established for uniform mesh and could be adapted to some non-uniform meshes obtained as the smooth image of a uniform grid, following the methodology of [3].

Another interesting question is to consider the fully discrete case of our problem, particularly due to the term $\partial_{x}^{2} \partial_{t}$, which mixes time and space. Perhaps a first attempt is just consider the time-discrete case

$$
\frac{u^{n+1}-u^{n}}{\Delta t}-\frac{\partial_{x}^{2} u^{n+1}-\partial_{x}^{2} u^{n}}{\Delta t}=p^{n+1} \partial_{x} u^{n+1}+q^{n+1} u^{n+1}, \quad n=0,1, \ldots
$$

which is a possible discretization in time.

\section{REFERENCES}

[1] L. Baudouin and S. Ervedoza, Convergence of an inverse problem for a 1-D discrete wave equation. SIAM J. Control Optim. 51 (2013) 556-598.

[2] T.B. Benjamin, J.L. Bona and J.J. Mahony, Model equations for long waves in nonlinear dispersive systems. Philos. Trans. Roy. Soc. London Ser. A 272 (1972) 47-78.

[3] F. Boyer, F. Hubert and J. Le Rousseau, Discrete Carleman estimates for elliptic operators and uniform controllability of semi-discretized parabolic equations. J. Math. Pures Appl. 93 (2010) 240-276.

[4] F. Boyer and J. Le Rousseau, Carleman estimates for semi-discrete parabolic operators and application to the controllability of semi-linear semi-discrete parabolic equations. Ann. Inst. Henri Poincaré Anal. Non Linéaire 31 (2014) 1035-1078.

[5] T. Carleman, Sur un problème d'unicité pur les systèmes d'équations aux dérivées partielles à deux variables indépendantes. Ark. Mat., Astr. Fys. 26 (1939) 9.

[6] P.L. da Silva and I.L. Freire, A geometrical demonstration for continuation of solutions of the generalised BBM equation. Monatshefte für Mathematik 194 (2021) 495-502.

[7] S. Ervedoza and F. de Gournay, Uniform stability estimates for the discrete Calderón problems. Inverse Probl. 27 (2011) 125012.

[8] X. Fu, Q. Lü and X. Zhang, Carleman estimates for second order partial differential operators and applications. SpringerBriefs in Mathematics. Springer, Cham (2019). A unified approach, BCAM SpringerBriefs.

[9] A.V. Fursikov and O.Y. Imanuvilov, Controllability of evolution equations. Vol. 34 of Lecture Notes Series. Seoul National University, Research Institute of Mathematics, Global Analysis Research Center, Seoul (1996).

[10] V. Hernández-Santamaría and P. González Casanova, Carleman estimates and controllability results for fully-discrete approximations of 1-d parabolic equations. Preprint arXiv:2012.02156 (2020).

[11] V. Isakov, Inverse source problems. Vol. 34 of Mathematical Surveys and Monographs. American Mathematical Society, Providence, RI (1990). 
[12] S. Micu, On the controllability of the linearized Benjamin-Bona-Mahony equation. SIAM J. Control Optim. 39 (2001) 16771696.

[13] T.N.T. Nguyen, Carleman estimates for semi-discrete parabolic operators with a discontinuous diffusion coefficient and applications to controllability. Math. Control Relat. Fields 4 (2014) 203-259.

[14] L. Rosier and B.-Y. Zhang, Unique continuation property and control for the Benjamin-Bona-Mahony equation on a periodic domain. J. Differ. Equ. 254 (2013) 141-178.

[15] M. Yamamoto, One unique continuation for a linearized Benjamin-Bona-Mahony equation. J. Inverse Ill-Posed Probl. 11 (2003) 537-543.

[16] X. Zhang and E. Zuazua, Unique continuation for the linearized Benjamin-Bona-Mahony equation with space-dependent potential. Math. Ann. 325 (2003) 543-582.

[17] C. Zheng, Inverse problems for the fourth order Schrödinger equation on a finite domain. Math. Control Relat. Fields 5 (2015) $177-189$.

[18] E. Zuazua, Propagation, observation, and control of waves approximated by finite difference methods. SIAM Rev. 47 (2005) $197-243$. 